\title{
Optical Design of Compact Linear Fresnel Reflector Systems
}

\author{
Jie Zhu*, Ziwei Chen \\ Department of Architecture and Built Environment, University of Nottingham, University \\ Park, Nottingham, NG7 2RD, UK
}

\begin{abstract}
Compact linear Fresnel reflector (CLFR) system employing multiple receivers is promising with better optical performance and cost effectiveness compared to linear Fresnel reflector (LFR) system, especially for applications with limited ground availabilities. Nevertheless, only few researches have been conducted to evaluate optical design and performance of the CLFR system. In this study, geometrical models for the CLFR system with flat mirrors and receivers are developed on the basis of polar orientation. A comparative study of concentration characteristics among the LFR, CLFR-complete and CLFR-hybrid systems is conducted based on numerical, ray tracing simulation and experimental results. In addition, optical design analyses of the CLFR-hybrid system are carried out from various design aspects. It is noteworthy that the mirror arrangement and focal length should be optimized for the CLFR-hybrid system with considerations of the associated geometrical characteristic and optical performance. For a small-scale CLFR-hybrid system with a solar field width of $2100 \mathrm{~mm}$ and a focal length of $1500 \mathrm{~mm}$, the geometrical concentration ratio of 15.14 and ground utilization ratio of 0.95 are achieved respectively. The findings demonstrate the feasibility of the CLFR-hybrid system with flat mirrors and polar orientation, which provide progress to the concentrated solar power technology.
\end{abstract}

Keywords: Compact linear Fresnel reflector; CLFR-hybrid; Geometrical modelling; Optical performance.

* Corresponding author. Tel: +44 1158466141. 
E-mail address: jie.zhu@ nottingham.ac.uk

\section{Nomenclature}

Symbols

$\begin{array}{ll}b_{n} & \text { Half width of a mirror element (mm) } \\ B & \text { Half length of a receiver (mm) } \\ d & \text { Gap between two consecutive mirrors in central alternating array (mm) } \\ D & \text { Gap between the last mirror in common tilting array and the first adjacent } \\ f & \text { Focal length (mm) } \\ k & \text { Utilization ratio } \\ N & \text { Total number of mirror elements } \\ W & \text { Total solar field width of a CLFR system (mm) } \\ W_{n} & \text { Width of a mirror element (mm) } \\ x_{n} & \text { Centre reference position of a mirror element (mm) }\end{array}$

Greek letters

$\alpha \quad$ Tilt angle of a mirror element $\left(^{\circ}\right)$

$\beta \quad$ Inclination angle of a receiver $\left(^{\circ}\right)$

$\Omega_{1} \quad$ Subtended angle by the sun (')

$\boldsymbol{\theta} \quad$ edge of receiver and proposed horizontal plane $\left(^{\circ}\right)$ 


\section{Subscripts}

c Central alternating mirror array of a CLFR-hybrid system

co Mirror array of a CLFR-complete system

1 Common tilting mirror array on the left side of a CLFR-hybrid system

Common tilting mirror array on the right side of a CLFR-hybrid system

Abbreviations

CLFR Compact linear Fresnel reflector

CR Geometrical concentration ratio

LFR Linear Fresnel reflector

PTC Parabolic trough collector

STC Solar tower concentrator 


\section{Introduction}

The supply shortage of conventional fossil fuels and environmental concern about climate changing lead to a great need to harness renewable energy for a sustainable future. Solar energy is regarded as the largest available carbon-neutral energy on the planet, which offers great potentials to be utilized by various technologies [1]. Besides direct conversion of the solar radiation into electricity with photovoltaic technology, the solar energy also can be transformed into thermal energy by solar collectors. Generally, solar collectors are categorized into non-concentrating and concentrating types. Non-concentrating solar collectors are commonly used in domestic hot water heating systems, whereas concentrating solar collectors are popular in thermal power generations with high efficiencies at medium to high operational temperatures, especially for locations receiving high solar beam radiation [2].

Based on the focus geometry, concentrating solar collectors are classified into parabolic tough concentrator (PTC), solar tower concentrator (STC), parabolic dish and linear Fresnel reflector (LFR) concentrator. Though PTC is a more mature and widely applied technology, its higher initial cost, complex manufacture process and serious challenge of wind resistance are noted [3]. Alternatively, the LFR system in a simple design is capable of harnessing the solar radiation efficiently with less land usage [4]. It has been proved to be a better choice for energy production where land availability is limited [5]. For the LFR system with a single centred receiver as illustrated in Fig. 1, each mirror element is suitably placed at a proposed tilt angle so that the incident solar radiation converges onto the receiver after reflection. 


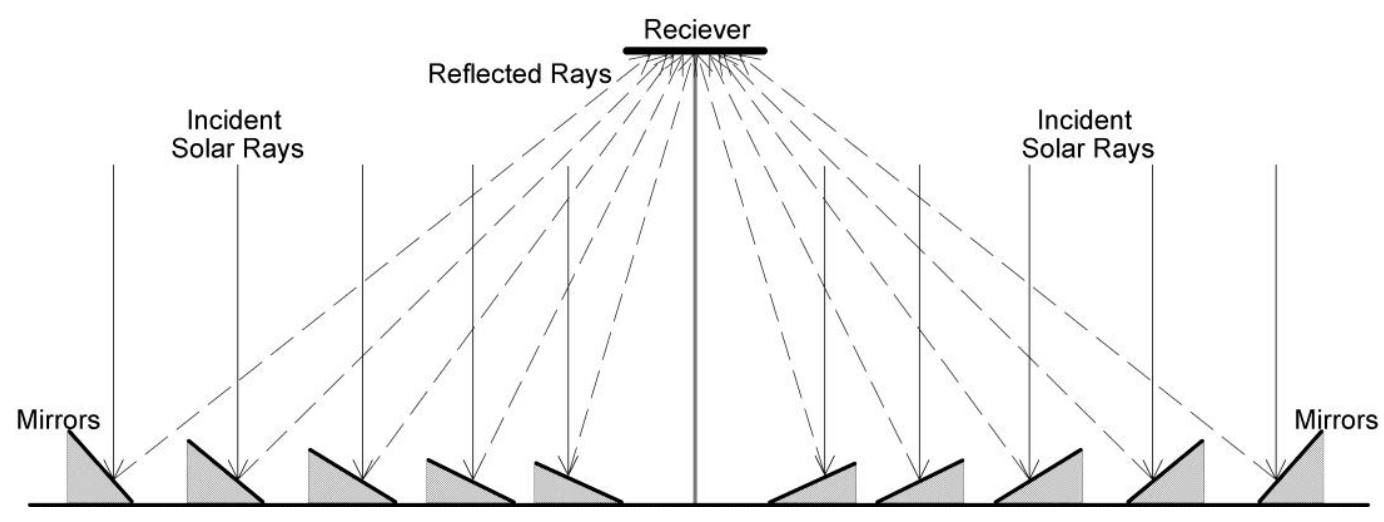

Fig. 1. Schematic diagram of LFR system

Optical designs of the LFR system have been investigated from different aspects, such as solar field layout and orientation, tracking algorithm and aiming strategy, reflector curvature and receiver configuration [6]. Among them, the solar field orientation influences significantly the other optical parameters. For instance, the complexity of a tracking mechanism depends on the orientation. Three common orientations are namely north-south, east-west and polar axis. Both north-south and east-west orientations are mostly preferred for applications, with which tracking schemes are based on the flat ground. In operation, reflectors follow the sun movement by varying their tilt angles under the control of a tracking system $[7,8]$. Consequently, end or lateral losses may occur when the reflected solar rays do not impinge on the receiver due to the longitudinal component of the solar radiation $[9,10]$. On the other hand, mirrors involved in the LFR system are commonly predefined with constant width and shift for the sake of simplicity. Such a simple optical design results in shading of the incoming solar radiation and blocking of the reflected solar radiation by adjacent mirror elements [11], which are illustrated in Fig. 2. Numerous researches have been conducted to minimize energy losses owing to the end, inter-row shading and blocking effects. For example, optical variables such as the width and position of each mirror element, gap between consecutive mirrors and receiver height have been considered in literatures [9, 10, 12, 13]. Abbas and Martínez-Val [13] observed an evident increase in the annual energy 
efficiency for a LFR system with mirrors of variable widths and shifts, but more complicated optical modelling and additional installation cost are required accordingly. On the other hand, the receiver has remarkable impacts on optical and thermal performances of the LFR system as well, which can be assembled horizontally, vertically or in triangular configuration [14]. To address the drawbacks of shading and blocking of the LFR system, Barlev, Vidu and Stroeve [1] suggested increasing receiver height, but the spacing between two adjacent mirror elements causes more ground usage and investment on the receiver tower. Contrarily, the system with polar axis orientation is more efficient with the simplest tracking system and the absorbed solar energy can be maximized while energy losses are minimized [15]. The polar orientated system has great adaptability to terrain with reduced installation requirement and cost. The simple polar mounted system usually adopts one single axis aligned to be roughly parallel to the axis of the earth rotation around the north and south poles. On the other hand, to ensure the solar field perpendicular to the solar rays throughout the year, various designs of the dual-axis solar tracker are proposed to follow the sun movement in both elevation and azimuth directions as reflectors and receiver are fixed at predefined tilt angles and positions $[16,17]$. Comparatively, dual-axis tackers are more promising for small-scale applications, whereas more structural considerations should be taken for large scale applications due to variable inclination angles of the entire solar field. Practically, the impacts of high slope and large solar field not only complicate the support structure required to elevate the array, but also increase the wind load which rises exponentially with height above the ground [18].

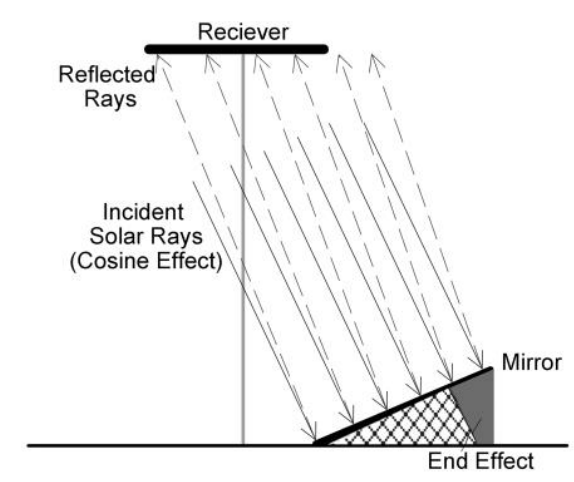




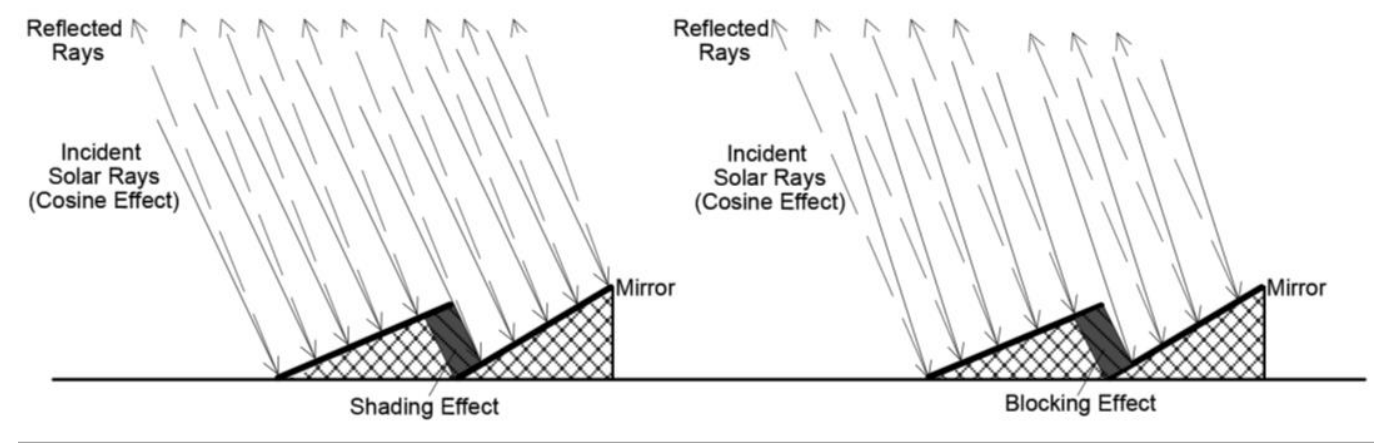

Fig. 2. Cosine, end, shading and blocking effects in LFR system

Alternatively, an innovative concept of compact linear Fresnel reflector (CLFR) system with multiple receivers is developed to minimize energy losses as depicted in Fig. 3. Different from the LFR system, one receiver is installed at each side of the solar field, which allows consecutive mirrors to redirect the sunlight to the two receivers respectively. The CLFR system has better system cost effectiveness [19] and is promising for applications with limited ground availabilities [1].

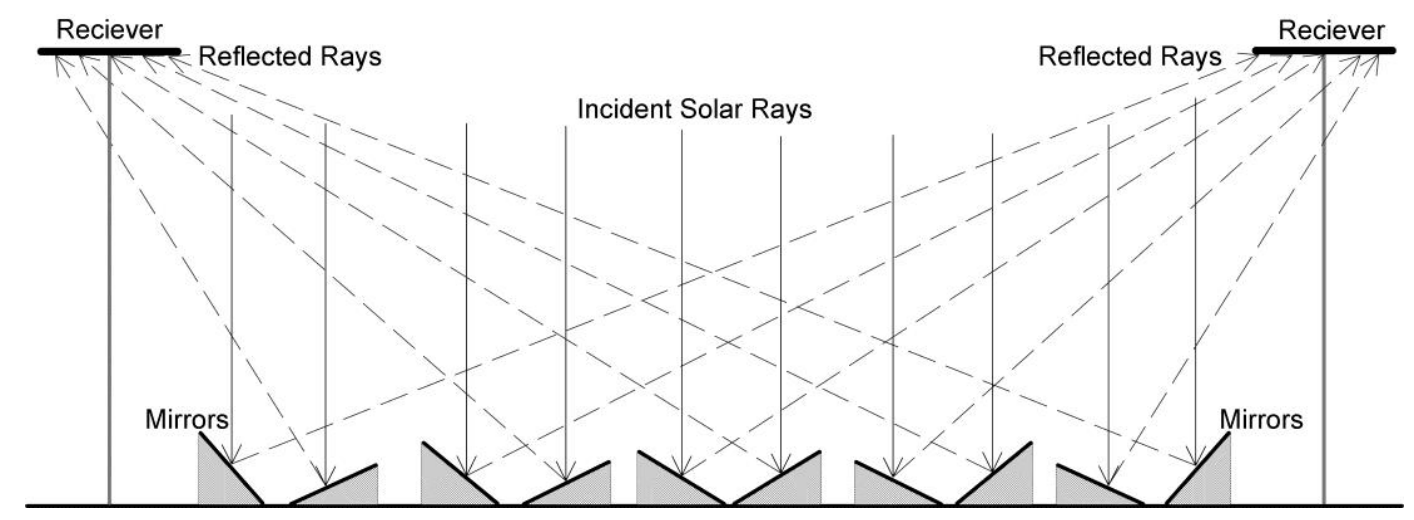

Fig. 3. Schematic diagram of CLFR system

The CLFR system has been investigated with limited optical designs and available demonstrations. Mills and Morrison [20] evaluated the CLFR concept applied for a largescale solar thermal electricity generation plant from various aspects, including the receiver orientation and structure, mirror field configuration and packing density. Chaves and Collares-Pereira [21] assessed variable mirror sizes and shapes for the CLFR system. In particular, Montes, Rubbia, Abbas and Martínez-Val [19] presented two CLFR 
configurations, as shown in Fig. 4. Mirrors are arranged with alternating tilts for pointing to one or the other receiver in the CLFR-complete configuration, while only mirrors located in the central solar field alternate their tilts in the CLFR-hybrid configuration. They observed that both CLFR configurations successfully minimize the blocking and shading losses, whereas lateral losses decrease the optical efficiency due to the great dispersion of reflected rays from mirrors located far from the receivers.

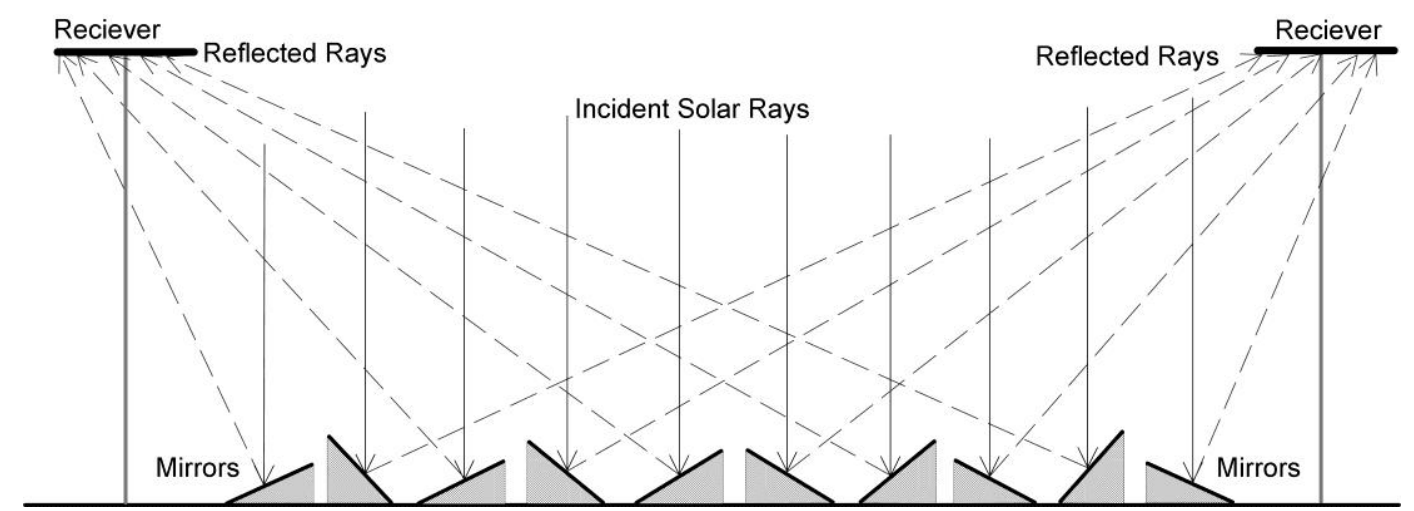

(a)

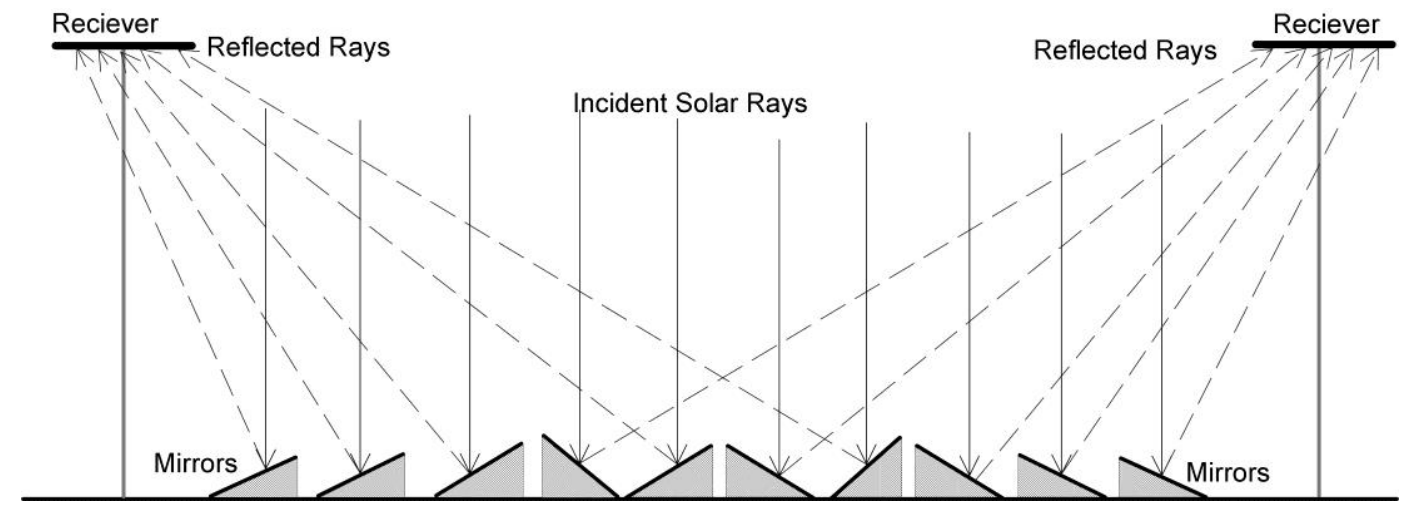

(b)

Fig. 4. Schematic diagrams of CLFR configurations (a) complete (b) hybrid

In terms of optical performance evaluations of the LFR and CLFR systems, studies have been conducted through numerical modelling [7, 9, 22-24], ray tracing simulation [10, 13, 25-27] and experiment [28-31]. For instance, Barale, Heimsath, Nitz and Toro [6] adopted a generalized methodology to optimize geometry for a LFR prototype built in Sicily, including optical modelling with ray tracing techniques and effect analyses of mirror and receiver 
geometries. On the other hand, Pino, Caro, Rosa and Guerra [29] simulated a LFR solar plant using optical and thermodynamic modelling, which was validated with operating data gathered from a supervisor system.

Researches in the optical design and performance evaluation of the CLFR system through simulation and experiment are limited in the literature. The investigations in the existing literature generally have been conducted for the CLFR system with north-south and east-west orientations, while the polar orientated CLFR system has not been concerned much. Furthermore, the optical performance comparison between the LFR and CLFR systems has been rarely mentioned in the literature, which would be desirable for realizing and promoting the CLFR system application. Thus, the optical design and performance evaluation of a small-scale CLFR system with polar orientation are carried out in this study. For the sake of simplicity and cost effectiveness, flat reflection mirrors are employed, which are made of glass. Besides, a comparative study of the concentration characteristics among the LFR, CLFR-complete and CLFR-hybrid configurations is performed through geometrical modelling, ray tracing simulation as well as experiment. To validate the geometrical models, experimental investigations are implemented with a small-scale test rig in Nottingham, UK. Through numerical simulation, optical design of the CLFR-hybrid system is assessed from different design aspects (e.g. mirror array arrangement, solar field area, focal length, receiver tilt angle, etc.) by evaluating associated geometrical parameters and optical performance.

\section{Geometrical modelling of CLFR system}

Geometrical modelling of the CLFR system is developed by referring to the methodology in literatures $[23,32]$. The modelling is based on polar orientation, flat mirrors are defined with variable widths and spacing to eliminate end, shading and blocking effects. Two flat receivers are designed to absorb the reflected solar rays with a given full width $(2 B)$ and an inclination 
angle $(\beta)$. In addition, the sun conical angle (represented by $2 \Omega_{1}=32^{\prime}$ ) is considered for more accurate optical design. The following assumptions are made:

(1) The system has a perfect tracking scheme to follow the sun movement;

(2) Only direct/beam solar radiation is considered.

(3) The sunlight rays are incident axially, which perfectly and normally collimate on mirrors in the solar field;

(4) Flat mirror elements are featured to be specularly reflecting.

\subsection{Common tilting mirror array modelling}

For the CLFR-hybrid system, the mirror arrangement of the common tilting array near the receivers is identical to that of the LFR system, while mirror elements in the central field are placed with alternating tilts to focus the sunrays on the respective receivers. The geometrical model for the common tilting array is established, which can be also applied for the LFR system. Regarding to axially symmetric feature of the CLFR-hybrid system, a basic geometry of the left-sided common tilting mirror array is illustrated in Fig. 5. The central point of each mirror element is positioned along the horizontal plane $\left(X X^{\prime}\right)$, the vertical $\mathrm{Z}$-axis passes through the central point of the flat receiver. Original point $(O)$ of the defined coordinate system is the intersectional point of the X-axis and Z-axis. In this study, focal length $(f)$ represents the height of the receiver lower edge over the horizontal plane. 


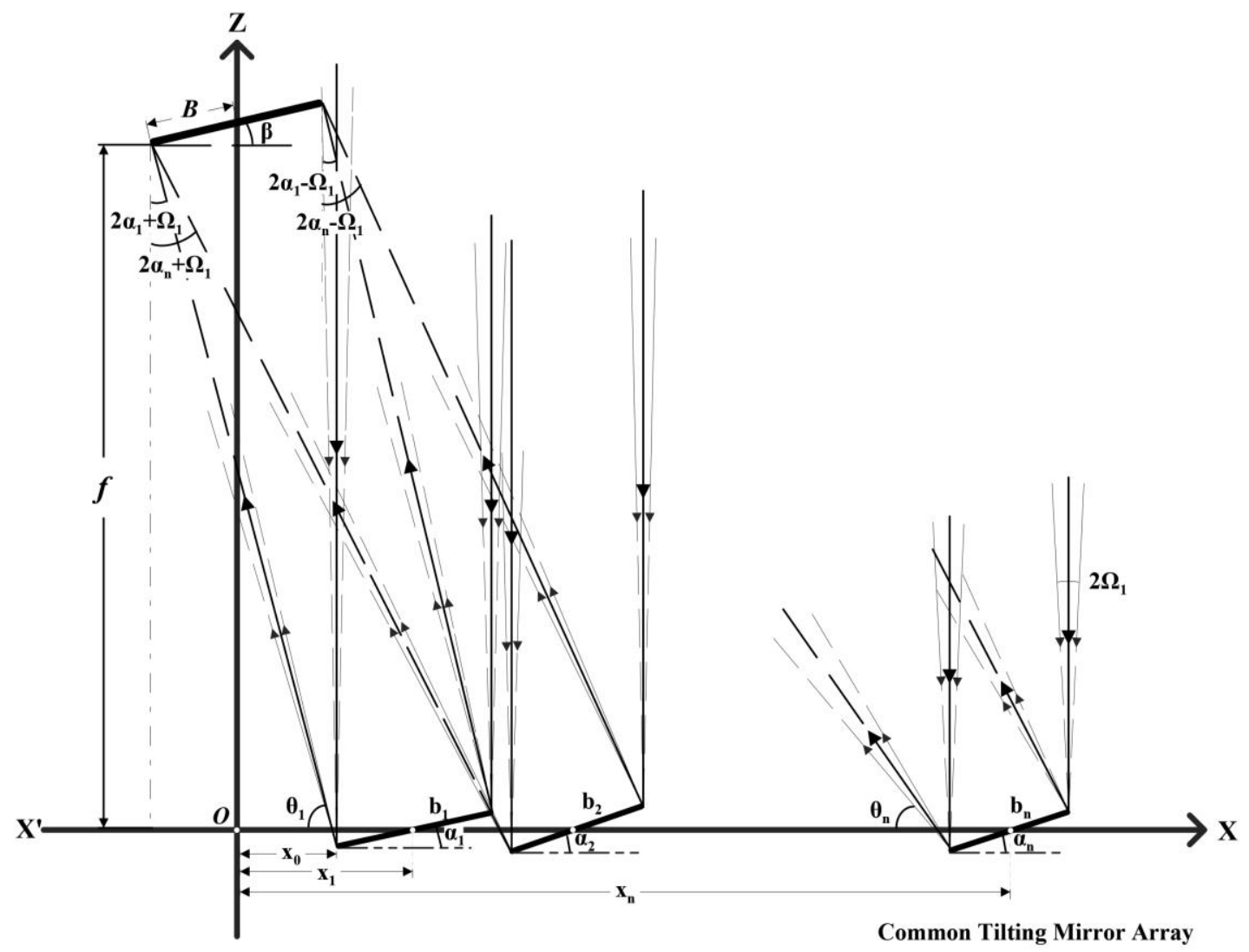

Fig. 5. Schematic diagram of common tilting mirror array of CLFR system

Each mirror element is characterized by three optical variables — width $\left(W_{n}=2 b_{n}\right)$, tilt angle $\left(\alpha_{n}\right)$, and reference position $\left(x_{n}\right)$ which is determined as the distance from the original point $(O)$ to the mirror element centre point. Theoretically, the tilt angle of a mirror element is proposed to ensure the extreme outer sunlight ray impinging on its lower edge strikes at the receiver lower edge after reflection, while the extreme outer ray impinging on the mirror upper edge strikes at the receiver upper edge. The angle between the reflected extreme outer solar ray impinging on the receiver lower edge and horizontal plane $X X^{\prime}$ is introduced as $\theta_{n}$. To avoid projection caused by the flat receiver, mirror elements are precluded underneath the receiver directly. Hence, the lower edge position of the first mirror element is determined as $x_{0}=B \cos \beta+(f+2 B \sin \beta) \cdot \tan \Omega_{1}$. Design variables of the first mirror element are given by:

$\theta_{1}=90^{\circ}-2 \alpha_{1}-\Omega_{1}$ 


$$
\begin{aligned}
& \tan \theta_{1}=\frac{f+b_{1} \sin \alpha_{1}}{x_{0}+B \cos \beta} \\
& \tan \left(2 \alpha_{1}-\Omega_{1}\right)=\frac{2 b_{1} \cos \alpha_{1}+(f+2 B \sin \beta) \cdot \tan \Omega_{1}}{f-b_{1} \sin \alpha_{1}+2 B \sin \beta} \\
& W_{1}=2 b_{1} \\
& \mathrm{x}_{1}=x_{0}+b_{1} \cos \alpha_{1}
\end{aligned}
$$

To eliminate shading and blocking problems, the sunlight rays impinging on the lower edge of the second mirror firstly touches the upper edge of the first mirror element and then strikes at the receiver lower edge after reflection. Following the similar logic, design variables of the $n_{\text {th }}$ mirror element are expressed as:

$\theta_{n}=90^{\circ}-2 \alpha_{n}-\Omega_{1}$

$\tan \theta_{n}=\frac{f+b_{n} \sin \alpha_{n}}{B \cos \beta+x_{n}-b_{n} \cos \alpha_{n}}$

$\tan \left(2 \alpha_{n}-\Omega_{1}\right)=\frac{x_{n}-B \cos \beta+b_{n} \cos \alpha_{n}}{f-b_{n} \sin \alpha_{n}+2 B \sin \beta}$

$\frac{2 b_{1}}{\sin \theta_{2}}=\frac{x_{2}-b_{2} \cos \alpha_{2}-x_{0}}{\sin \left(180^{\circ}-\theta_{2}-\alpha_{1}\right)}$ If $n=2$

$\frac{2 b_{n-1}}{\sin \theta_{n}}=\frac{x_{n}-b_{n} \cos \alpha_{n}-x_{n-1}+b_{n-1} \cos \alpha_{n-1}}{\sin \left(180^{\circ}-\theta_{n}-\alpha_{n-1}\right)}$ If $n>2$

\subsection{Central alternating mirror array modelling}

A schematic diagram of the CLFR-hybrid system for modelling the central alternating mirror array is presented in Fig. 6. The total solar field width $(W)$ of the CLFR-hybrid system is defined as the distance between the two receiver centre points. The gap between the last 
mirror element in the common tilting array and first adjacent alternating mirror is represented as $D$, and the gap of two consecutive mirror elements with opposite tilting directions in the alternating array is defined as $d$. According to different optical design requirements, $D$ and $d$ can be adjusted properly. To avoid shading and blocking effects, there are two different mirror arrangements depending on the lower edge positions of two adjacent mirrors. One case is that the extreme outer solar ray impinging on the prior mirror element's lower edge touches the lower edge of the latter mirror element and finally strikes at the receiver's lower edge after reflection (e.g. see the arrangement of the first and second mirrors in the alternating array with $b_{\mathrm{c} 1} \sin \alpha_{\mathrm{c} 1} \geq b_{\mathrm{c} 2} \sin \alpha_{\mathrm{c} 2}$ in Fig. 6). The other one is that the extreme outer solar ray impinging on the latter mirror's lower edge touches the lower edge of the prior mirror element and strikes at the receiver's lower edge after reflection (e.g. see the arrangement of the third and fourth mirrors in the alternating array with $b_{\mathrm{c} 3} \sin \alpha_{\mathrm{c} 3}<b_{\mathrm{c} 4} \sin \alpha_{\mathrm{c} 4}$ in Fig. 6). 


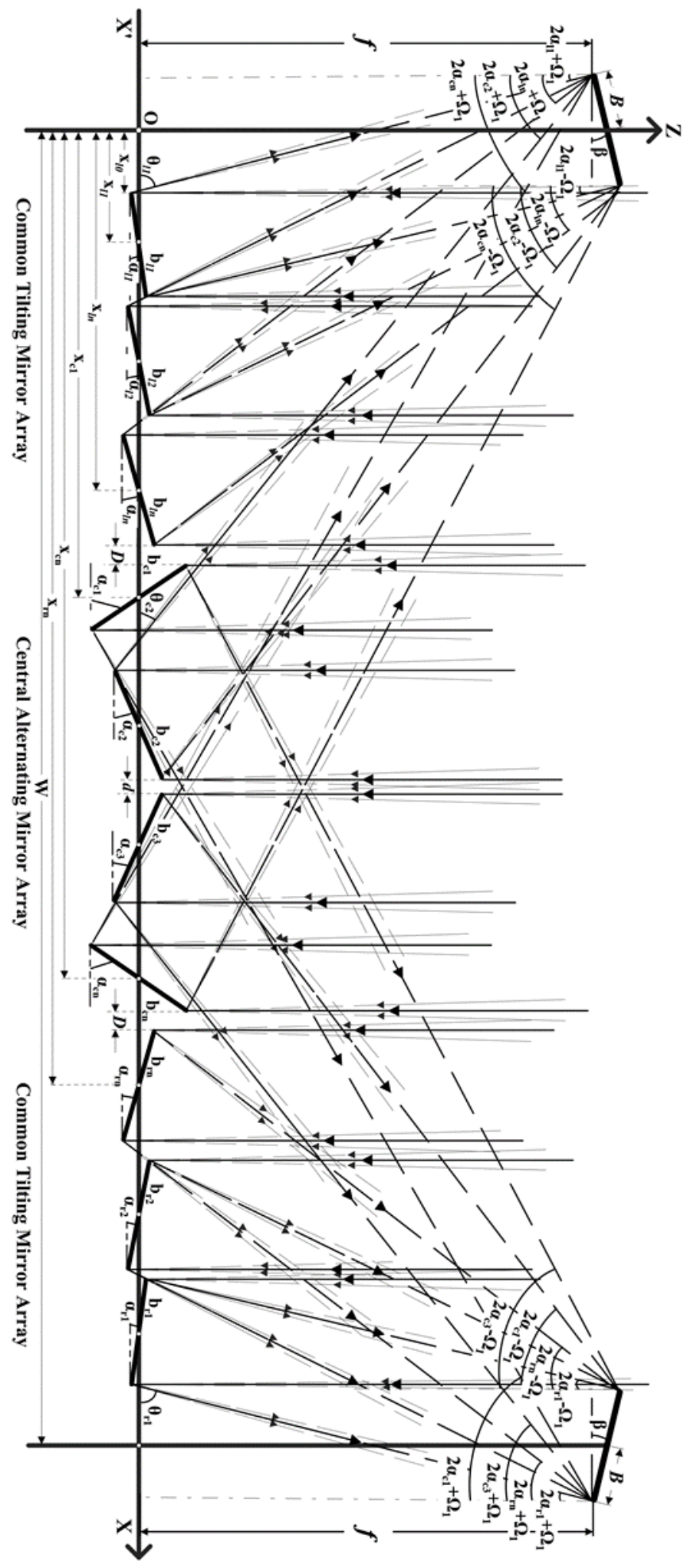

Fig. 6. Schematic diagram of CLFR-hybrid system 
The upper edge position of the last mirror element in the left tilting array is given as $x_{l o}=x_{l n}+b_{l n} \cos \alpha_{l n}$. Consequently, the associated variables of the first alternating mirror element are given by:

$\theta_{\mathrm{c} 1}=90^{\circ}-2 \alpha_{\mathrm{c} 1}-\Omega_{1}$

$\tan \theta_{\mathrm{c} 1}=\frac{f+b_{\mathrm{c} 1} \sin \alpha_{\mathrm{c} 1}}{W-\left(x_{\mathrm{lo}}+D+2 b_{\mathrm{c} 1} \cos \alpha_{\mathrm{c} 1}\right)+B \cos \beta}$

$\tan \left(2 \alpha_{\mathrm{c} 1}-\Omega_{1}\right)=\frac{W-\left(x_{\mathrm{lo}}+D\right)}{f-b_{\mathrm{c} 1} \sin \alpha_{\mathrm{c} 1}+2 B \sin \beta}$

In the central alternating solar field, the even and odd mirror elements have different features as they redirect the solar rays to two respective receivers. Design variables of the even mirror elements are defined as:

$\theta_{\mathrm{cn}}=90^{\circ}-2 \alpha_{\mathrm{cn}}-\Omega_{1}$

$\tan \left(\theta_{\mathrm{cn}}\right)=\frac{f+b_{\mathrm{cn}} \sin \alpha_{\mathrm{cn}}}{x_{\mathrm{cn}}-b_{\mathrm{cn}} \cos \alpha_{\mathrm{cn}}+B \cos \beta}$

$\tan \left(2 \alpha_{\mathrm{cn}}-\Omega_{1}\right)=\frac{x_{\mathrm{cn}}+b_{\mathrm{cn}} \cos \alpha_{\mathrm{cn}}-B \cos \beta}{f-b_{\mathrm{cn}} \sin \alpha_{\mathrm{cn}}+2 B \sin \beta}$

For the case that the prior mirror's lower edge is lower than that of the latter one (i.e. $\left.b_{\mathrm{c}(\mathrm{n}-1)} \sin \alpha_{\mathrm{c}(\mathrm{n}-1)} \geq b_{\mathrm{cn}} \sin \alpha_{\mathrm{cn}}\right)$,

$\tan \left(\theta_{\mathrm{c}(\mathrm{n}-1)}\right)=\frac{b_{\mathrm{c}(\mathrm{n}-1)} \sin \alpha_{\mathrm{c}(\mathrm{n}-1)}-b_{\mathrm{cn}} \sin \alpha_{\mathrm{cn}}}{\left(x_{\mathrm{cn}}-b_{\mathrm{cn}} \cos \alpha_{\mathrm{cn}}\right)-\left(x_{\mathrm{c}(\mathrm{n}-1)}+b_{\mathrm{c}(\mathrm{n}-1)} \cos \alpha_{\mathrm{c}(\mathrm{n}-1)}\right)}$

For the other case that the prior mirror's lower edge is higher than that of the latter one (i.e. $\left.b_{\mathrm{c}(\mathrm{n}-1)} \sin \alpha_{\mathrm{c}(\mathrm{n}-1)}<b_{\mathrm{cn}} \sin \alpha_{\mathrm{cn}}\right)$, 


$$
\tan \left(\theta_{\mathrm{cn}}\right)=\frac{b_{\mathrm{cn}} \sin \alpha_{\mathrm{cn}}-b_{\mathrm{c}(\mathrm{n}-1)} \sin \alpha_{\mathrm{c}(\mathrm{n}-1)}}{\left(x_{\mathrm{cn}}-b_{\mathrm{cn}} \cos \alpha_{\mathrm{cn}}\right)-\left(x_{\mathrm{c}(\mathrm{n}-1)}+b_{\mathrm{c}(\mathrm{n}-1)} \cos \alpha_{\mathrm{c}(\mathrm{n}-1)}\right)}
$$

On the other hand, variables of the odd mirror elements are given as:

$$
\begin{aligned}
& \theta_{\mathrm{cn}}=90^{\circ}-2 \alpha_{\mathrm{cn}}-\Omega_{1} \\
& \tan \left(\theta_{\mathrm{cn}}\right)=\frac{f+b_{\mathrm{cn}} \sin \alpha_{\mathrm{cn}}}{W-\left(x_{\mathrm{c}(\mathrm{n}-1)}+b_{\mathrm{c}(\mathrm{n}-1)} \cos \alpha_{\mathrm{c}(\mathrm{n}-1)}+d+2 b_{\mathrm{cn}} \cos \alpha_{\mathrm{cn}}\right)+B \cos \beta} \\
& \tan \left(2 \alpha_{\mathrm{cn}}-\Omega_{1}\right)=\frac{W-\left(x_{\mathrm{c}(\mathrm{n}-1)}+b_{\mathrm{c}(\mathrm{n}-1)} \cos \alpha_{\mathrm{c}(\mathrm{n}-1)}+\mathrm{d}\right)-B \cos \beta}{f-b_{\mathrm{cn}} \sin \alpha_{\mathrm{cn}}+2 B \sin \beta} \\
& x_{\mathrm{cn}}=x_{\mathrm{c}(\mathrm{n}-1)}+b_{\mathrm{c}(\mathrm{n}-1)} \cos \alpha_{\mathrm{c}(\mathrm{n}-1)}+d+b_{\mathrm{cn}} \cos \alpha_{\mathrm{cn}}
\end{aligned}
$$

Furthermore, the developed model of the central alternating mirror array can be also applied for the CLFR-complete configuration by defining the upper edge position of the first mirror element on the left side as $x_{\mathrm{co}}=B \cos \beta+(f+2 B \sin \beta) \cdot \tan \Omega_{1}$.

\subsection{Evaluation metrics}

In this study, optical performances of the LFR and CLFR systems are mainly evaluated by three factors, namely geometrical concentration ratio, ground utilization ratio and solar illumination on the receiver surface. Theoretically, for the system with horizontally orientated flat receiver(s), the geometrical concentration ratio $(C R)$ is defined as the ratio of the total mirror field area to the receiver surface area [33]. For the LFR system, it is expressed as

$$
C R_{\mathrm{LFR}}=\frac{\sum_{n=1}^{N} W_{n} \cos \alpha_{n}}{2 B}
$$

Where $N$ is total number of mirror elements. 
For the CLFR-hybrid system, $C R$ is given by

$C R_{\text {CLFR-hybrid }}=\frac{\sum_{l n=1}^{N_{l n}} W_{l n} \cos \alpha_{l n}+\sum_{r n=1}^{N_{r n}} W_{r n} \cos \alpha_{r n}+\sum_{c n=1}^{N_{c n}} W_{c n} \cos \alpha_{c n}}{4 B}$

Where subscripts $l n$ and $r n$ indicate mirror elements in the left-sided and right-sided common tilting arrays respectively and $c n$ represents an alternating mirror in the central solar field.

For the CLFR-complete system, $C R$ is defined as

$C R_{\text {CLFR-complete }}=\frac{\sum_{n=1}^{N} W_{n} \cos \alpha_{n}}{4 B}$

The ground utilization ratio $(k)$ implies the ratio of the total mirror field area to the total solar field area. For the LFR system, it is given by

$k_{\mathrm{LFR}}=\frac{\sum_{n=1}^{N} W_{n} \cos \alpha_{n}}{2 \cdot\left(x_{n}+b_{n} \cos \alpha_{n}\right)-2 B}$

Where $x_{n}+b_{n} \cos \alpha_{n}$ represents half width of the solar field of the LFR system.

For the CLFR-hybrid and CLFR-complete systems, $k$ can be determined as

$k_{\text {CLFR-hybrid }}=\frac{\sum_{l n=1}^{N_{l n}} W_{l n} \cos \alpha_{l n}+\sum_{r n=1}^{N_{r m}} W_{r n} \cos \alpha_{r n}+\sum_{c n=1}^{N_{c n}} W_{c n} \cos \alpha_{c n}}{W-2 B}$

$k_{\text {CLFR-complete }}=\frac{\sum_{n=1}^{N} W_{n} \cos \alpha_{n}}{W-2 B}$ 


\section{Modelling approach}

Matlab is used to solve the developed geometrical models in Section 2, and then the mirror element profile including the width, tilt angle and reference position for a given system can be produced accordingly. To investigate solar radiation distributions on receiver surfaces, ray tracing simulation is implemented with the advanced TracePro program. The ray tracing simulation is capable of verifying the defined tilt angles and shifts of mirrors whether lateral, shading and blocking problems occur. A flow chart showing the whole simulation process in this study is presented in Fig. 7. 


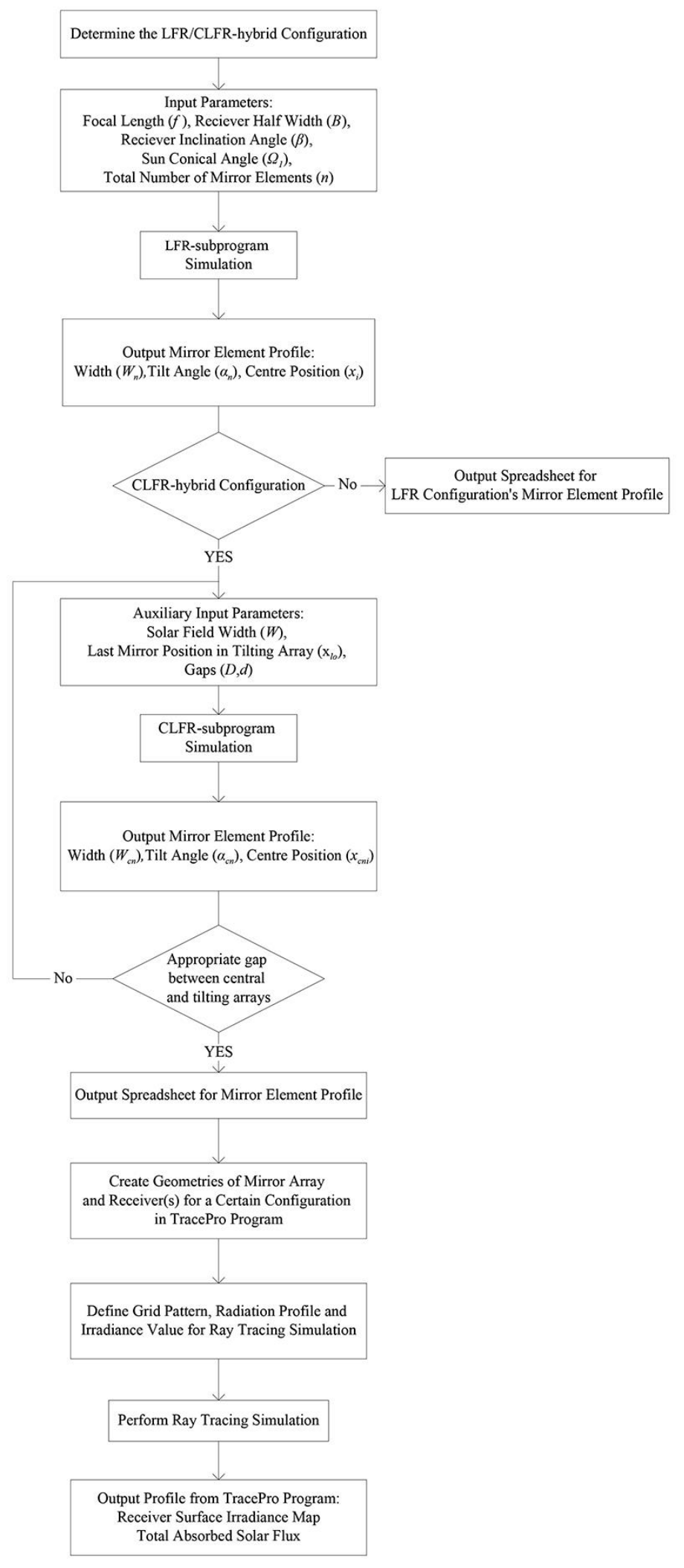

Fig. 7. Flow chart of simulation process with Matlab and TracePro program 
In TracePro program, flat mirrors are assigned with reflectance of $95 \%$ and receivers are defined with absorptance of 0.9 . For simulation, the solar field is divided into a large number of equal divisions and in each division, a cone contains a certain number of generated uniform solar rays, which are all defined to be normally impinged onto the solar field [32]. The solar irradiance value as the input parameter in the radiation profile is determined in accordance with the local weather condition. In such way, the absorbed solar radiation value could be validated with the experimental data under the same weather condition.

\section{Experiment set up}

\subsection{Experiment description}

A small-scale CLFR test rig is designed and constructed in Nottingham (latitude of $52.97^{\circ}$ and longitude of $\left.1.18^{\circ}\right)$. Specifications of the test rig are presented in Table 1 . The test rig has an overall solar field area of $2.10 \mathrm{~m}^{2}$, which is feasible to track the sun movement freely. Due to the small-scale set up, narrow flat mirrors and horizontally placed flat receivers are employed.

Table 1. Specifications of the CLFR test rig

\begin{tabular}{|l|l|}
\hline \multicolumn{2}{|l|}{ Solar Field } \\
\hline Solar Field Width & $2100 \mathrm{~mm}$ \\
\hline Solar Field Length & $1000 \mathrm{~mm}$ \\
\hline Focal Length Range & $1000 \mathrm{~mm}-2000 \mathrm{~mm}$ \\
\hline Mirror and Receiver Profile & \\
\hline Mirror Material & Glass \\
\hline Mirror Element Length & $1000 \mathrm{~mm}$ \\
\hline Mirror Element Thickness & $5 \mathrm{~mm}$ \\
\hline Receiver Width & $64 \mathrm{~mm}$ \\
\hline Receiver Length & $1000 \mathrm{~mm}$ \\
\hline Receiver Inclination Angle & $0^{\circ}$ \\
\hline
\end{tabular}


As shown in Fig. 8, the test rig mainly comprises a base framework, a solar field platform with supporting beams, saw-tooth shaped bases, mirror elements and receivers with flexible supporting structures. For the polar orientation, the inclination angle and position of the solar field platform relate to the solar altitude and azimuth at a specific time. Hence, the supporting beams are employed to provide an accurate inclination angle for the solar field platform. A series of flat mirror strips are placed onto the saw-tooth shaped bases at the proposed positions and tilt angles, which are obtained from the theoretical modelling.

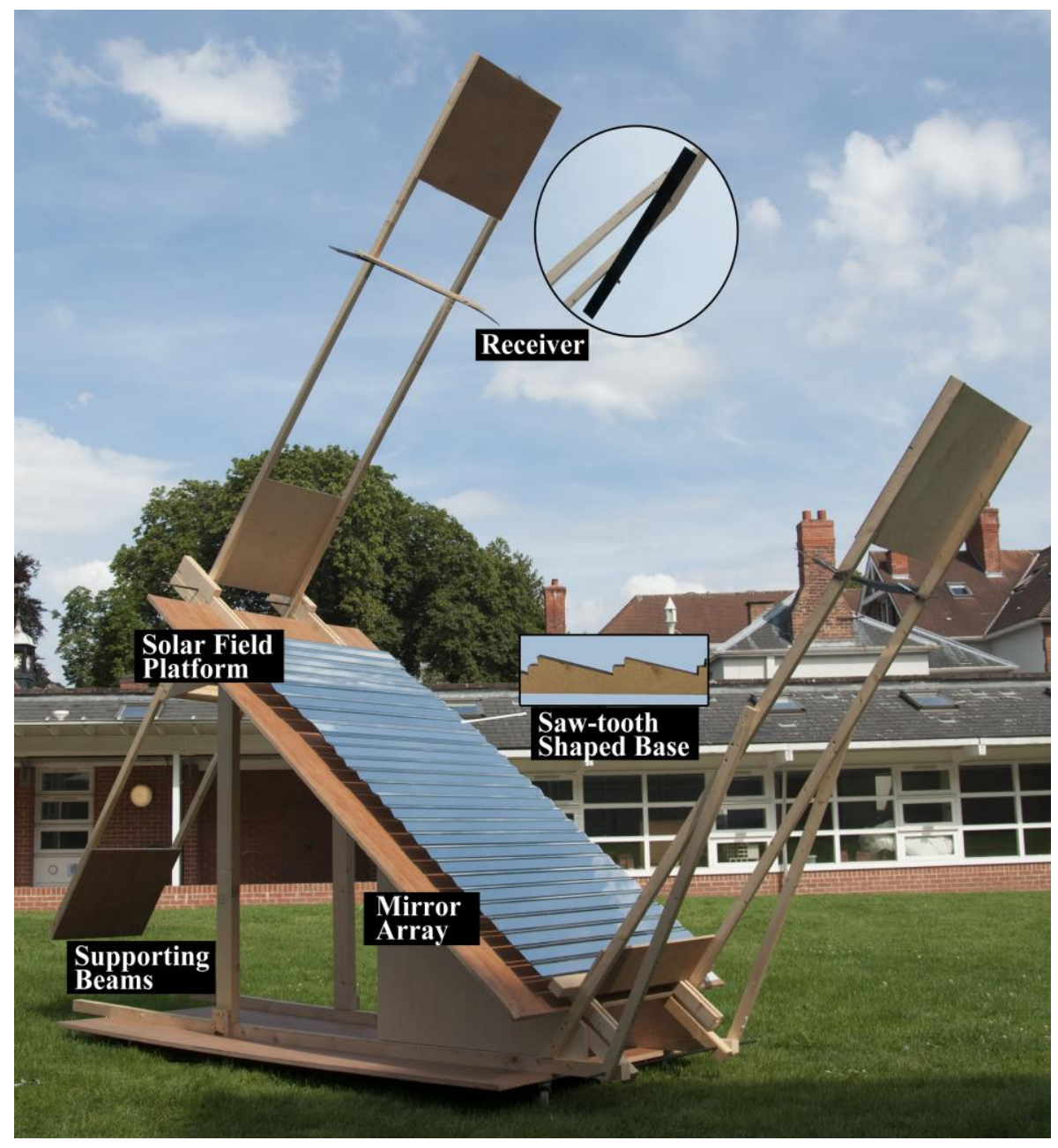

Fig. 8. Photo of the CLFR test rig

\subsection{Flux measurement}

The main objective of the experiment is to obtain the total received solar intensity on receiver surfaces. To achieve this, flux measurement is required either using a direct or indirect 
measuring method. Calorimeter and flux sensor are the common direct measuring instruments to provide flux value on a target, while an indirect device is capable of providing a continuous flux map. Digital camera is mostly preferred as the indirect measuring instrument by offering good resolution. For a good quality digital camera, a sensor is made up of millions of small photosensitive sensors (pixels) and outputs a voltage directly relating to the amount of photons which strike it [34]. The amount of photons hitting each pixel is directly proportional to the flux incident on a target. In this study, a Nikon D90 digital camera is used to produce irradiance maps on receiver surfaces. Reference images of receiver surfaces are taken under different irradiance levels for calibration. Additionally, a pyranometer is employed to record the solar radiation level during the experimental period. In terms of data processing, the image processing toolbox in Matlab is used to obtain the received solar flux.

\section{Results and discussion}

\subsection{Optical performances of LFR and CLFR}

\subsubsection{Mirror array arrangement}

Regarding to operability of a small-scale system with polar orientation, the solar field with a width of $2100 \mathrm{~mm}$ and a length of $1000 \mathrm{~mm}$ is adopted for the LFR, CLFR-hybrid and CLFRcomplete systems at a focal length of $1500 \mathrm{~mm}$. In addition, three arrangements are proposed for the CLFR-hybrid configuration with different ratios of the common tilting mirrors to the alternating ones. As listed in Table 2, the total number of mirror elements varies greatly for different configurations. With the same solar field width and focal length, the CLFRcomplete system accommodates 56 pieces of flat mirrors, while the LFR system only allows 42 pieces to be placed in the solar field. It is also seen that with fewer mirrors in the common tilting array, more mirrors could be added in the alternating array. The results imply that the 
alternating array requires less ground area, which contributes to a more densely packed array arrangement for the CLFR system.

Table 2. Total number of mirror elements for LFR, CLFR-hybrid and CLFR-complete configurations

\begin{tabular}{|c|c|c|c|c|c|}
\hline \multirow{2}{*}{ Configuration } & \multicolumn{2}{|c|}{ Number of Mirrors } & Total Number of \\
\cline { 2 - 6 } & & $\begin{array}{c}\text { Common Tilting } \\
\text { Arrays }\end{array}$ & $\begin{array}{c}\text { Alternating } \\
\text { Array }\end{array}$ & $\begin{array}{c}\text { Percentage of } \\
\text { Alternating Mirrors }\end{array}$ \\
\hline \multicolumn{2}{|c|}{ LFR } & 42 & 0 & 42 & $0 \%$ \\
\hline \multirow{2}{*}{$\begin{array}{c}\text { CLFR- } \\
\text { hybrid }\end{array}$} & A & 14 & 36 & 50 & $72 \%$ \\
\cline { 2 - 6 } & B & 22 & 24 & 46 & $52 \%$ \\
\hline \multicolumn{2}{|c|}{$\begin{array}{c}\text { CLFR- } \\
\text { complete }\end{array}$} & 28 & 16 & 44 & $36 \%$ \\
\hline
\end{tabular}

\subsubsection{Concentration and utilization ratios}

Optical performances of the above five configurations are shown in Fig. 9. By comparison, the CLFR-hybrid configurations have relatively higher concentration and utilization ratios, which are in consistent with the findings in literature [19]. Though the CLFR-complete system consists of more mirror elements, its concentration ratio is lower than those of the three CLFR-hybrid configurations owing to the large proportion of narrower mirrors located far from the respective targeted receivers. Theoretical results reveal that the CLFR-hybrid system could be a favourable alternative to the LFR system with higher concentration and ground usage ratios. On the other hand, the combination of mirror elements placed in the common tilting and alternating arrays influences not only the geometrical aspect but also optical performance. Among the CLFR-hybrid A, B, C systems, the most densely packed CLFR-hybrid A system with comparatively fewer mirrors in the common tilting array has the best optical performance, the geometrical concentration ratio of 15.14 and ground utilization ratio of 0.95 are obtained respectively. 


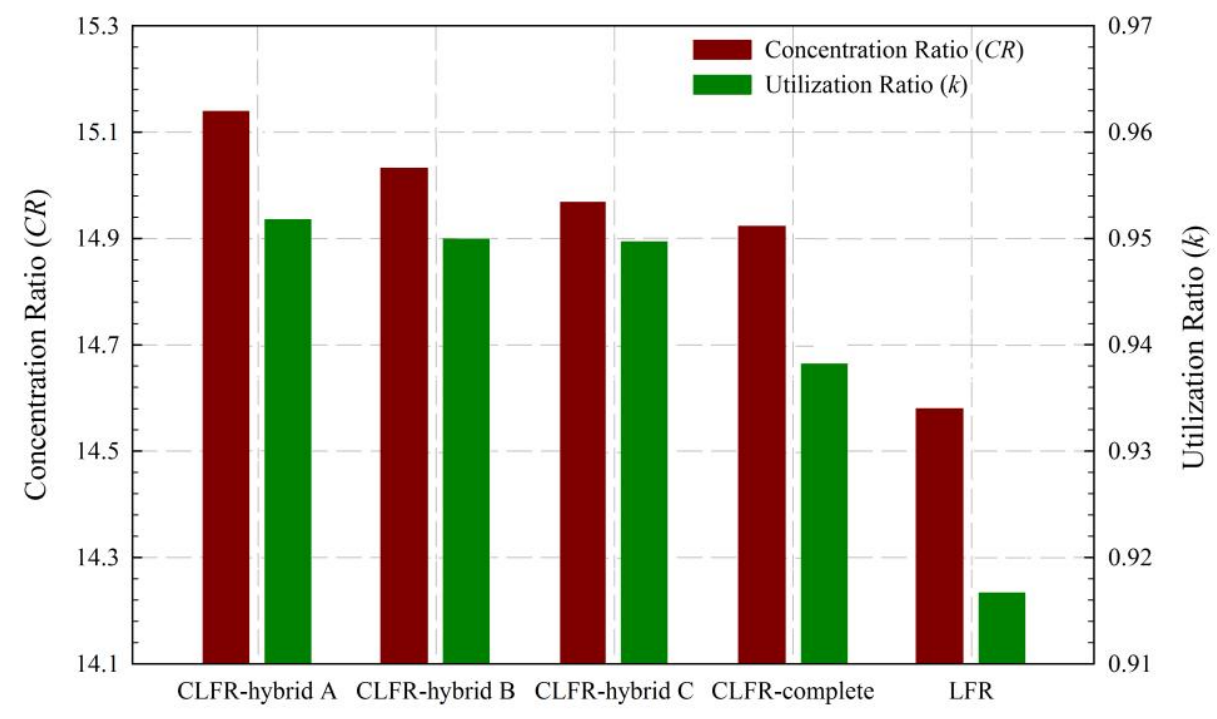

Fig. 9. Concentration and utilization ratios of LFR, CLFR-hybrid and CLFR-complete configurations

\subsubsection{Received solar energy}

On the basis of geometrical modelling, experimental investigations are conducted along with ray tracing simulation to figure out the solar illuminance on receiver surfaces. The total received solar energy on receiver surfaces obtained from experiment and ray tracing simulation are presented in Fig. 10. Based on the measurement data from the pyranometer, the average solar radiation during testing is approximately $600 \mathrm{~W} / \mathrm{m}^{2}$. Therefore, the input solar irradiance value in TracePro program is in accordance with the testing weather condition, which is also $600 \mathrm{~W} / \mathrm{m}^{2}$. It is observed that the variation pattern of simulation results matches with that of the experimental data. The simulation results indicate that $1150 \mathrm{~W}$ of the solar energy is received by the CLFR-hybrid A system, whereas $1055 \mathrm{~W}$ is obtained from the experiment. With respect to the energy efficiency of the CLFR system, it is defined as the ratio of the total received energy on receiver surfaces to the total incident energy on mirrors [19]. Referring to the experimental data, the energy efficiency of the CLFR-hybrid A system is approximately $91.3 \%$. 


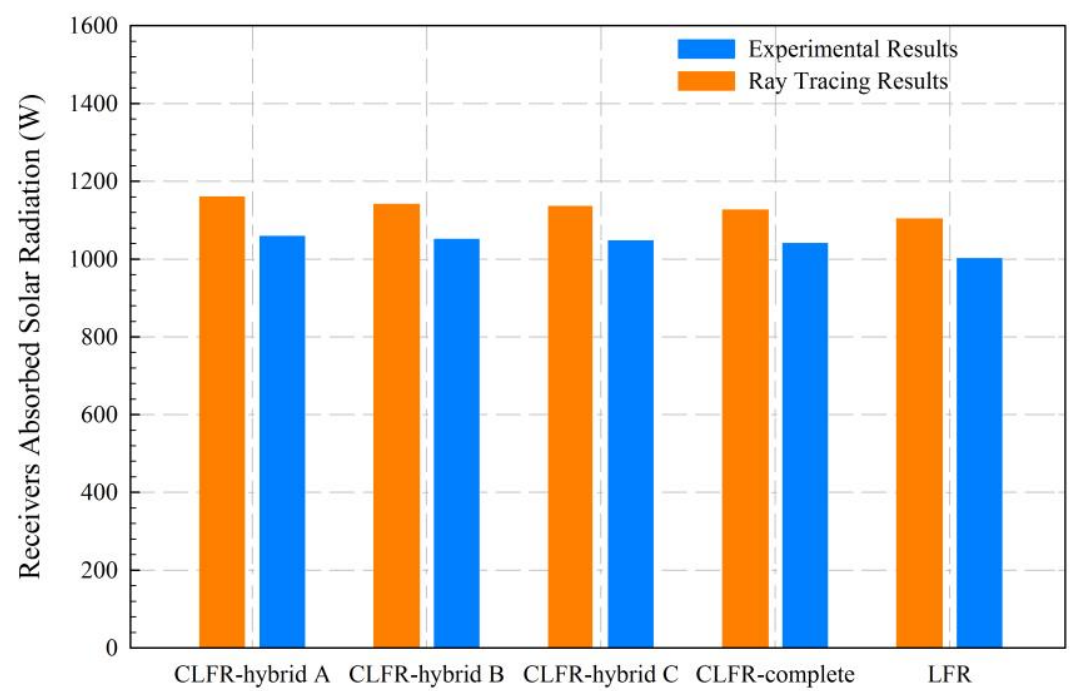

Fig. 10. Simulation and experimental results of received solar energy for LFR, CLFR-hybrid and CLFR-

complete configurations

The main reasons caused differences between the simulation and experimental results are clarified. Firstly, mirrors and receivers are given with assumed ideal properties (i.e. mirror reflectance of 0.95 and receiver absorptance of 0.9) in TracePro program. However, for the experimental set up, the reflectance of mirrors and absorptance of flat receivers differ from the defined values. Secondly, though the system with polar orientation tracks the sun movement freely, tracking errors may happen during the experiment, for example inaccurate positioning of the test rig. Thirdly, the digital camera captures illuminance images on receiver surfaces, which may be also influenced by the surroundings. Moreover, errors may occur in the image processing with Matlab including the calibration with reference flux images.

\subsection{Optical design for CLFR-hybrid system with limited solar field area}

Analysis is conducted to evaluate various optical designs of the CLFR-hybrid system from different design aspects, including the limited solar field area, total number of mirrors, focal length and receiver inclination angle. Based on the previous analysis, the CLFR-hybrid A configuration is chosen to assess the effect of focal length on the optical performance while 
the solar field width and length are set as $2100 \mathrm{~mm}$ and $1000 \mathrm{~mm}$ respectively. The gaps $D$ and $d$ are accordingly adjusted for the focal length varying from $1100 \mathrm{~mm}$ to $1900 \mathrm{~mm}$.

\subsubsection{Mirror variables}

The mirror profile of the CLFR system is evaluated based on the geometrical modelling results. The mirror width variations of the CLFR-hybrid A configuration at different focal lengths are presented in Fig. 11. Generally, the farther a mirror element is placed from its targeted receiver, the narrower width and larger tilt angle. However, the mirror width variation is more significant at a smaller focal length. In other words, much wider and narrower mirror elements are required for receivers located at a lower position. For instance, mirror width varies from $53.7 \mathrm{~mm}$ to $20.2 \mathrm{~mm}$ at a focal length of $1100 \mathrm{~mm}$, while the variation is from $46.3 \mathrm{~mm}$ to $25.2 \mathrm{~mm}$ at a focal length of $1900 \mathrm{~mm}$. Similar findings of the geometrical aspect have been noted by Mathur, Kandpal and Negi [32] as well.

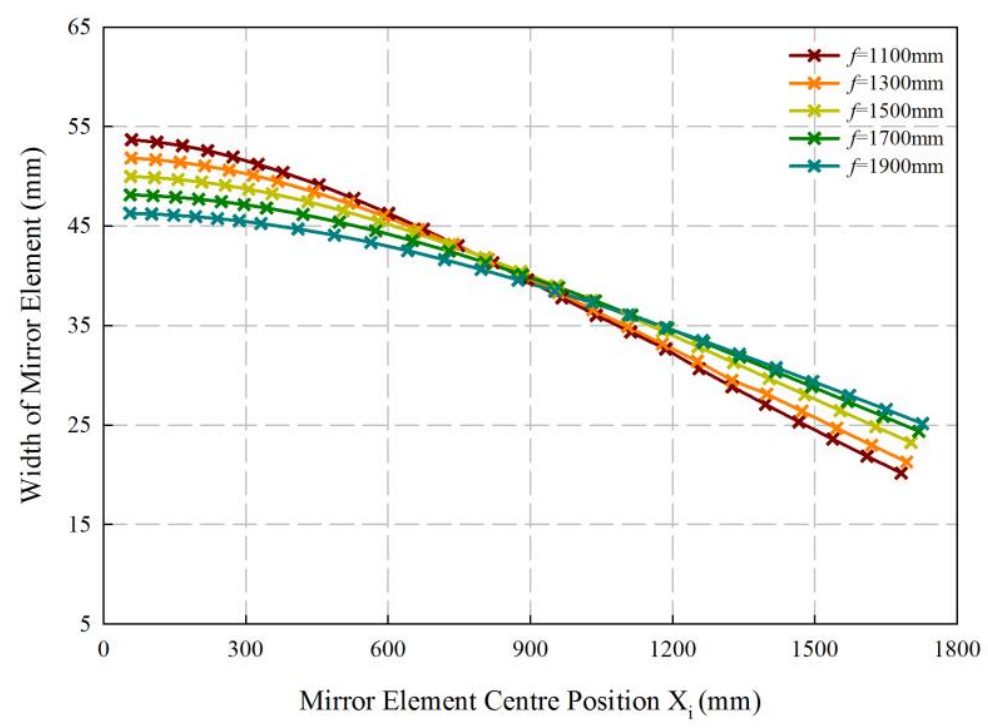

Fig. 11. Mirror width variations for CLFR-hybrid A configuration at different focal lengths

\subsubsection{Optical performance}


With regard to optical performance, the numerical results presented in Fig. 12 show that the focal length influences both concentration and ground utilization ratios as the solar field area is limited to a given value and there exists an optimum focal length. Both ratios gradually increase as the focal length rises from $1100 \mathrm{~mm}$ to $1500 \mathrm{~mm}$, and decrease with a further increase in the focal length. For the investigated solar field area of $2.10 \mathrm{~m}^{2}$, the optimum focal length for the CLFR-hybrid A configuration is $1500 \mathrm{~mm}$, at which both concentration and utilization ratios reach the maximum values.

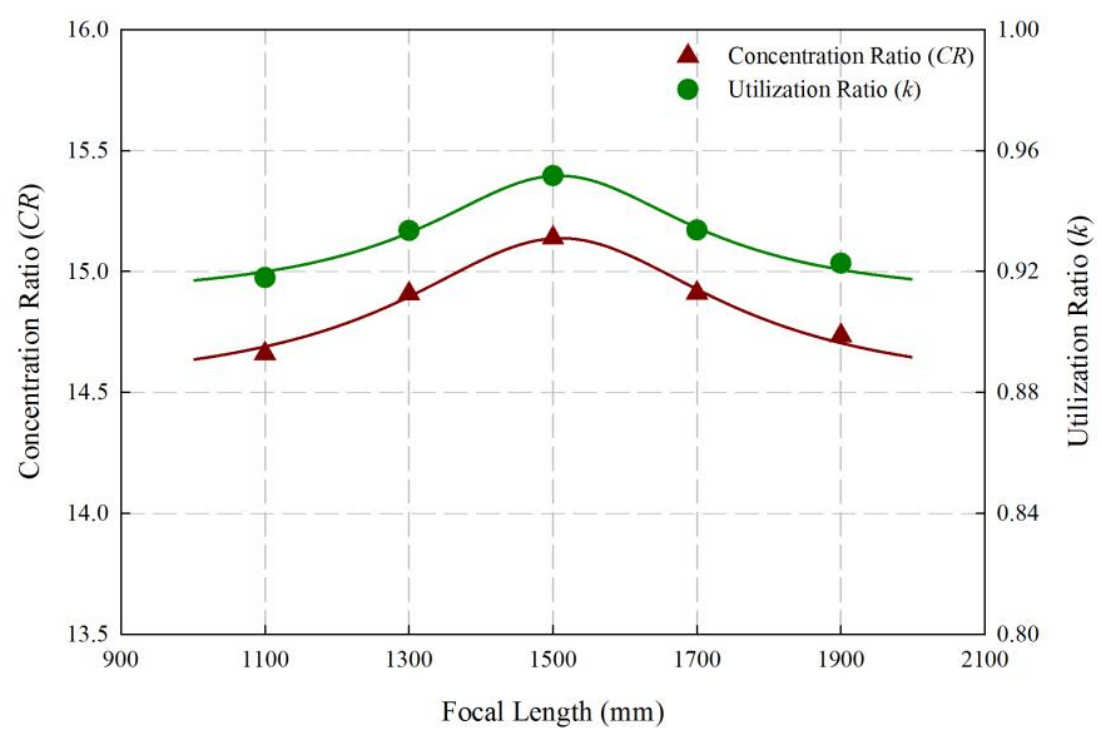

Fig. 12. Variations of concentration and utilization ratios with focal length for CLFR-hybrid A configuration

Specifically, variations of concentration and utilization ratios throughout the solar field of the CLFR-hybrid A configuration with a focal length of $1500 \mathrm{~mm}$ are shown in Fig. 13. The concentration ratio increases gradually with the mirror element position, while the utilization ratio decreases conversely due to the increasing shift of mirror elements toward the solar field rim. As the solar field width is limited to $2100 \mathrm{~mm}$, the gap variables ( $D$ and $d$ ) in the central alternating array are defined accordingly, which may be larger than the gaps in the common tilting array. This leads to the notable drop in the utilization ratio as the mirror element moves from the common tilting array to the central alternating array. 


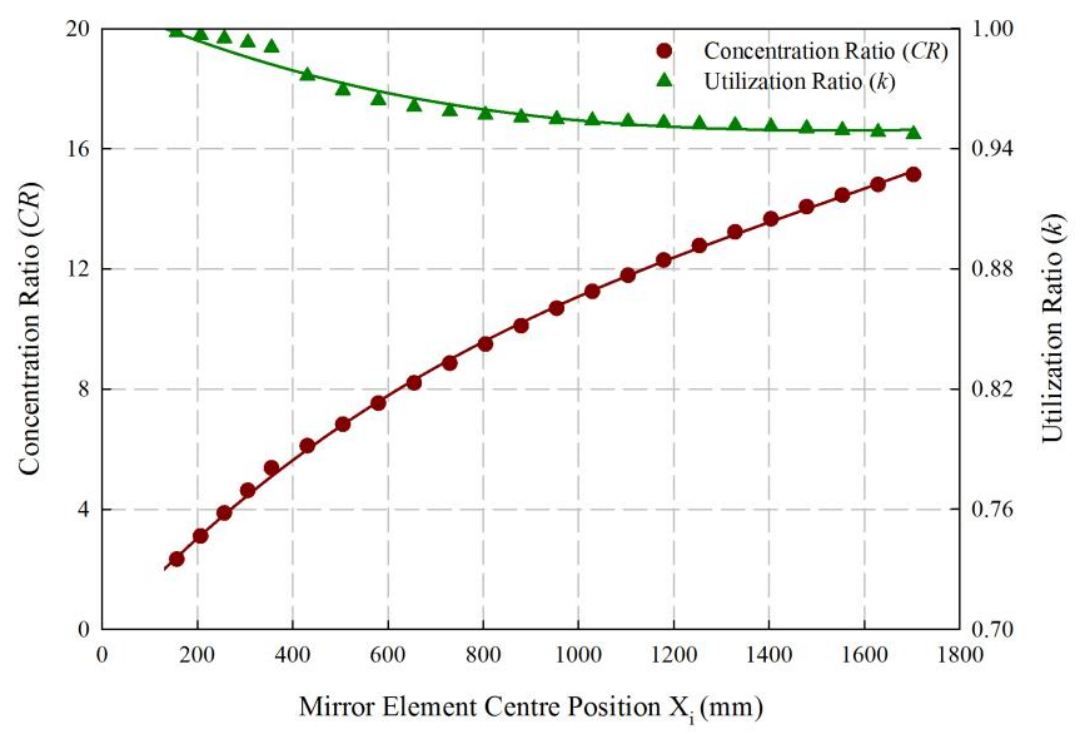

Fig. 13. Variations of concentration and utilization ratios for CLFR-hybrid A system with a focal length of $1500 \mathrm{~mm}$ and solar field width of $2100 \mathrm{~mm}$

Experimental data for the CLFR-hybrid A configuration at different focal lengths are shown in Fig. 14. The focal length has a comparatively more significant impact on the optical performance than the mirror arrangement. The results also indicate that the focal length should be optimized for optical design of the CLFR-hybrid system with a limited ground area to achieve better optical performance.

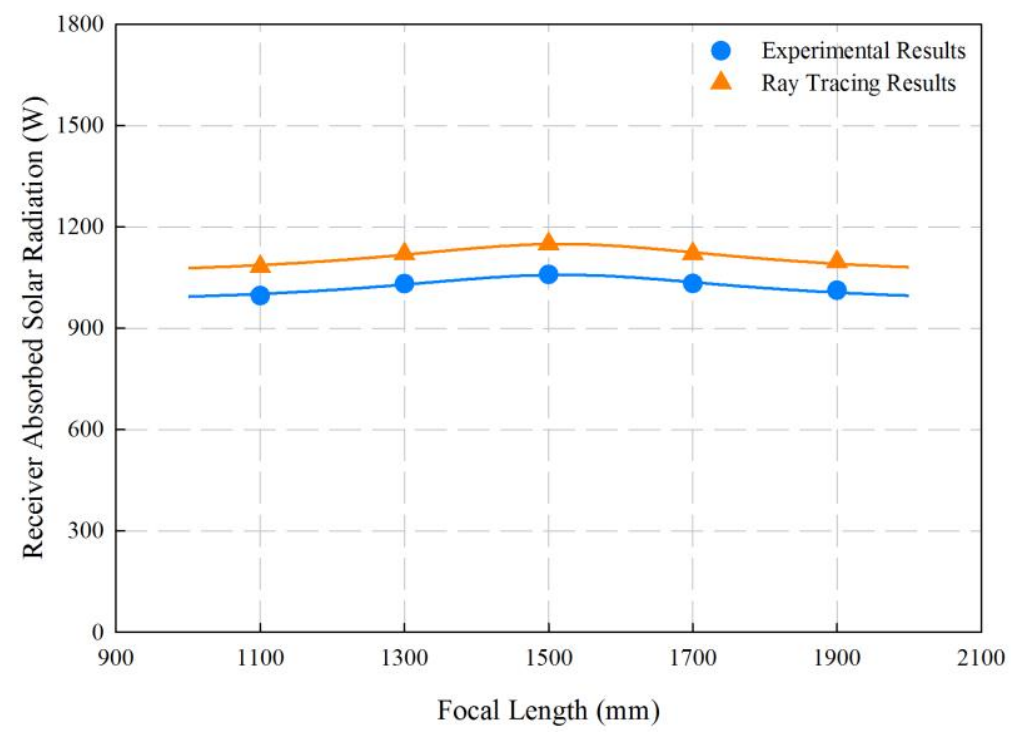

Fig. 14. Simulation and experimental results of received solar energy for CLFR-hybrid A configuration 


\subsubsection{Illuminance map}

Ray tracing simulation is then implemented to verify the proposed optical design on the basis of the geometrical modelling and produce the illuminance map. The ray tracing simulation of the CLFR-hybrid A system with a solar field width of $2100 \mathrm{~mm}$ and a focal length of $1500 \mathrm{~mm}$ is presented in Fig. 15. It can be evidently seen that the receivers on both sides receive all reflected rays from the proposed mirror array.

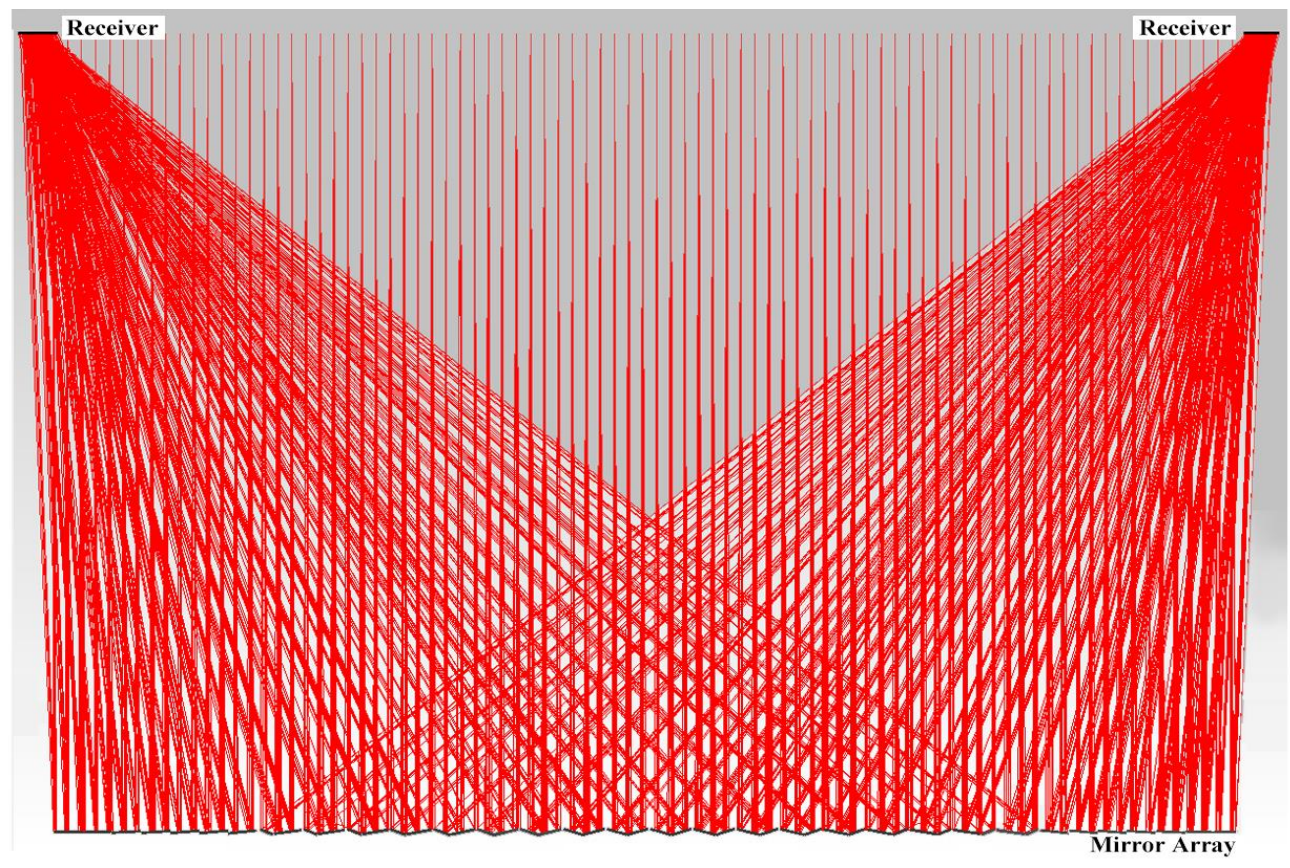

Fig. 15. Ray tracing simulation result of CLFR-hybrid A system

For the sake of simplicity, solar radiation distributions on receiver surfaces are obtained with only one mirror element through both experiment and ray tracing simulation, and illuminance maps are presented in Fig. 16. It is observed that almost the entire receiver surface is fully illuminated by the reflected solar rays from the mirror element, which is different from that of the CLFR system with point focusing Fresnel lens. The solar radiation distribution across the receiver surface from the experimental image is not well uniform owing to its uneven pattern. Comparatively, more uniform distribution on the receiver surface can be found in the TracePro illuminance map except for the boundary regions. This is due to the fact that the total number of generated solar rays for tracing is limited in TracePro program, and the 
generated rays from the defined solar source could not perfectly strike at the edges of each mirror element.

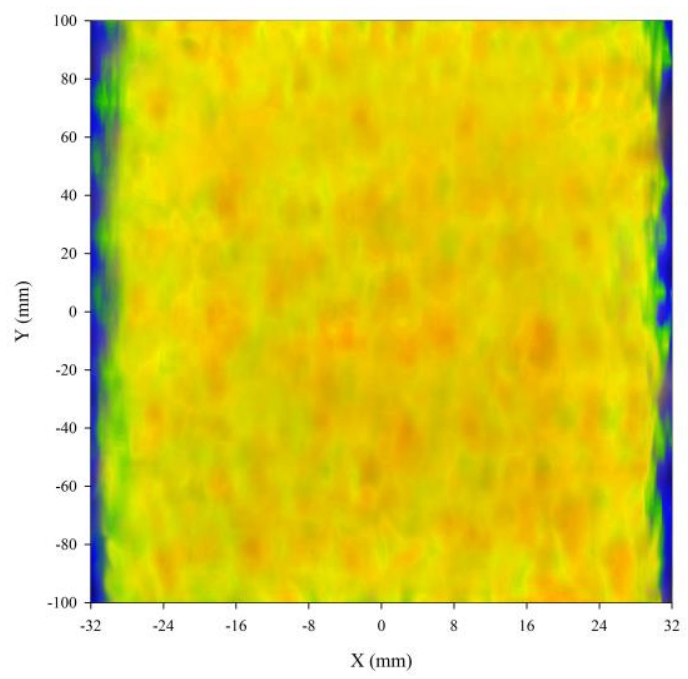

(a)

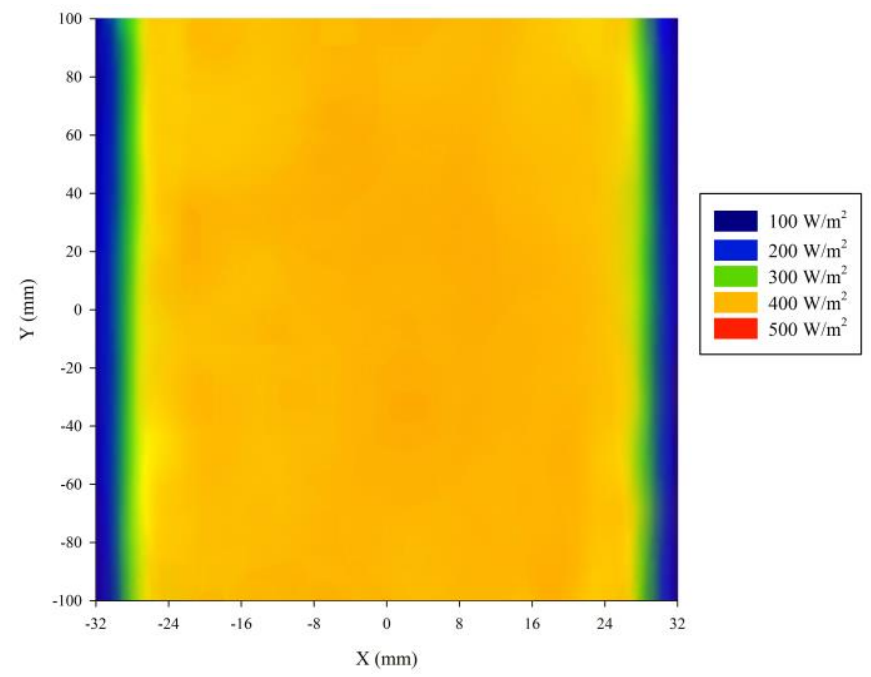

(b)

Fig. 16. Illuminance maps of receiver surface for CLFR-hybrid A system (a) experimental (b) ray tracing simulation results

\subsection{Optical design for CLFR-hybrid system with limited mirrors}

Besides the design limitation of the solar field area, the total number of mirror elements could be another restriction. Investigations are carried out for optical design of the CLFR-hybrid system with the fixed total number of mirrors as the focal length and solar field area vary accordingly to accommodate mirrors adequately. As presented in Table 3, to ensure 50 mirrors properly placed in the solar field, only a slight variation in the solar field width is observed while the focal length reduces from $2800 \mathrm{~mm}$ to $700 \mathrm{~mm}$.

Table 3. Solar field widths for CLFR-hybrid configurations with the same total number of mirror elements

\begin{tabular}{|c|c|c|c|}
\hline \multicolumn{2}{|c|}{ Configuration } & Solar Field Width (mm) & Focal Length (mm) \\
\hline \multirow{4}{*}{ CLFR-hybrid } & D & 1900 & 2800 \\
\cline { 2 - 4 } & E & 2000 & 2400 \\
\cline { 2 - 4 } & F & 2050 & 2000 \\
\cline { 2 - 4 } & A & 2100 & 1500 \\
\cline { 2 - 4 } & G & 2050 & 1100 \\
\cline { 2 - 4 } & H & 1950 & 700 \\
\hline
\end{tabular}


Variations of concentrating and utilization ratios for the CLFR-hybrid configurations with the focal length are shown in Fig. 17. It is worth noting that the CLFR-hybrid A system with a focal length of $1500 \mathrm{~mm}$ and the largest solar field area is the optimum solution for accommodating 50 mirror elements. The concentrating ratio increases as the focal length rises from $700 \mathrm{~mm}$ to $1500 \mathrm{~mm}$, it reaches the maximum value of 15.14 as receivers are located at a height of $1500 \mathrm{~mm}$, and then decreases as the focal length continues to increase. Similarly, the utilization ratio reaches the maximum value of 0.95 at a focal length of $1500 \mathrm{~mm}$. Thus, the results imply the necessity to identify the optimum focal length and solar field area for the CLFR-hybrid system when the mirror array arrangement is defined with limited total number of mirror elements.

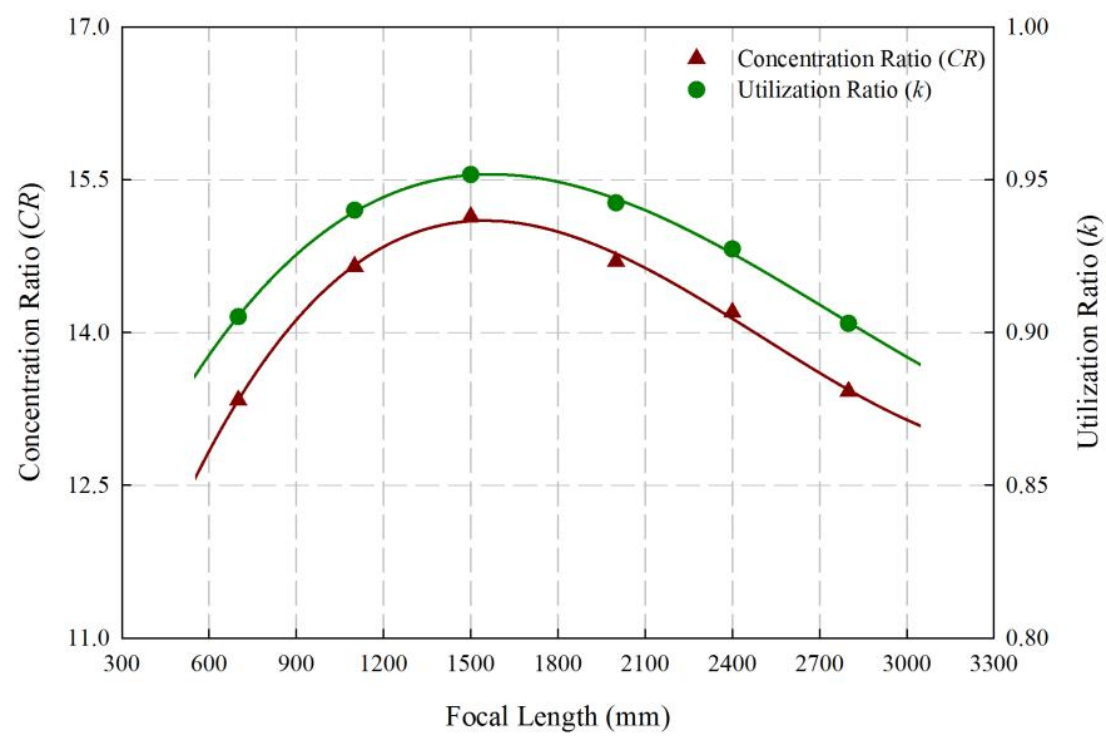

Fig. 17. Variations of concentration and utilization ratios with focal length for CLFR-hybrid configurations with 50 mirrors

\subsection{Optical design for CLFR-hybrid system at a given focal length}

Investigations are then conducted to figure out the optimum arrangements for the solar field width ranging from $1500 \mathrm{~mm}$ to $5000 \mathrm{~mm}$ as the focal length is fixed at $1500 \mathrm{~mm}$. As listed in Table 4, the proportion of mirrors in the central alternating array varies for different 
configurations. For a larger solar field area, fewer mirrors could be accommodated in the central alternating array while more mirrors are required in the common tilting array to achieve a higher concentration ratio. For instance, around $63 \%$ of total number of mirrors are placed in the central alternating array for the smallest solar field with a width of $1523 \mathrm{~mm}$, while only $5 \%$ of mirrors are in the central alternating field for the largest solar field with a width of $4997 \mathrm{~mm}$. On the other hand, a wider solar field leads to a greater variation range in mirror width at the given focal length. Therefore the number of narrow mirrors should also be taken into consideration, as extreme narrow mirrors located far from the receivers could not be accepted for practical application.

Table 4. Mirror arrangements of the CLFR-hybrid system at a fixed focal length of $1500 \mathrm{~mm}$

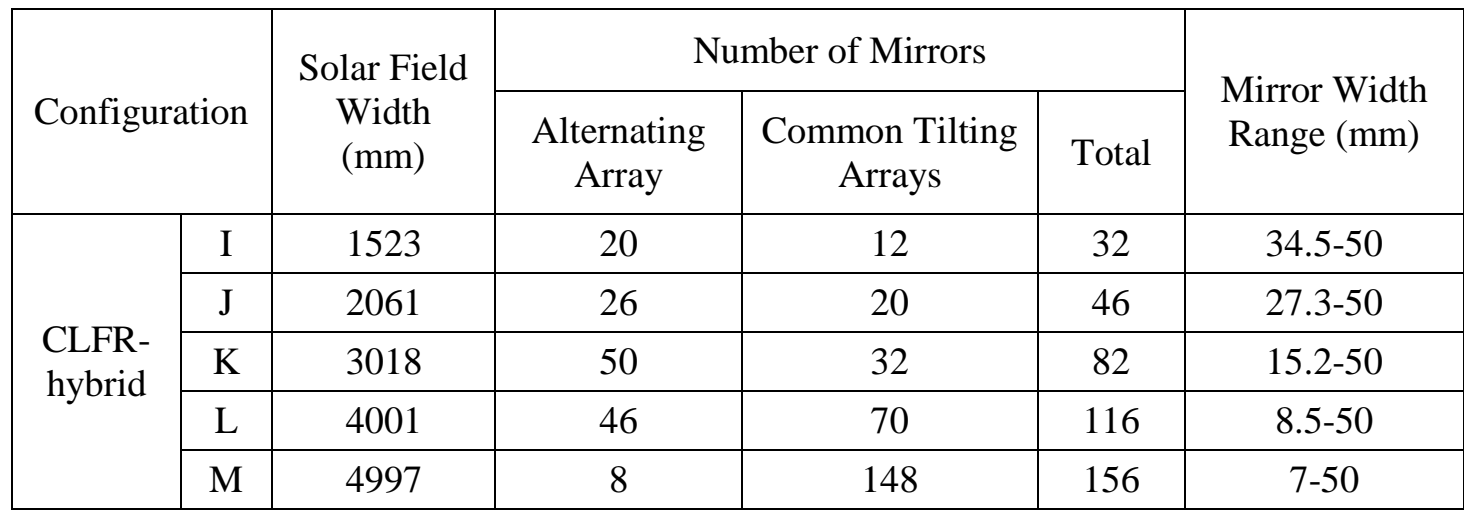

Variations of concentration and utilization ratios with the solar field width are illustrated in Fig. 18. It can be clearly seen that a larger solar field with more mirror elements leads to a higher concentration ratio. However, in order to avoid the shading and blocking problems, the total gap between adjacent mirrors increases accordingly with the solar field width, which results in a lower utilization ratio. Consequently, a comprehensive procedure should be followed to derive an optimal design of the CLFR-hybrid system, by taking the mirror array arrangement, focal length, concentration characteristic and ground usage into account. 


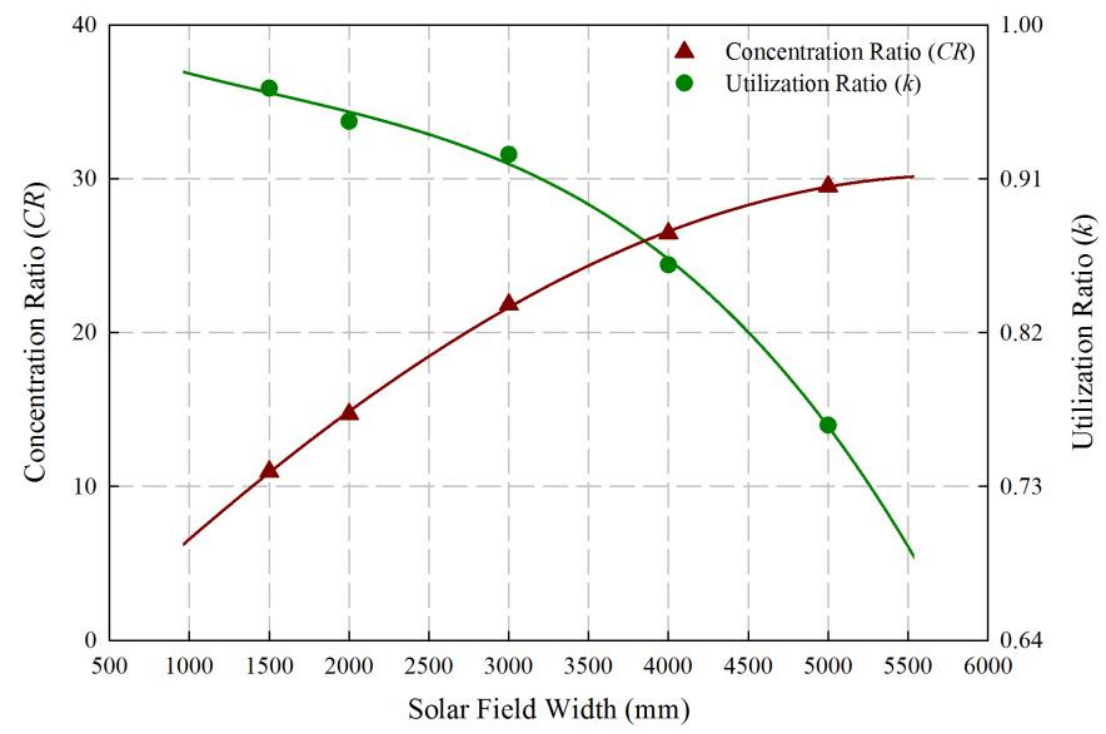

Fig. 18. Variations of concentration and utilization ratios with solar field width for CLFR-hybrid configurations at a focal length of $1500 \mathrm{~mm}$

\subsection{Optical design for CLFR-hybrid system with variable receiver inclination angles}

Previous investigations are conducted for the CLFR-hybrid system with horizontally oriented flat receivers, nevertheless flat receivers can be placed at an inclination angle. To evaluate the effect of receiver inclination angle on the optical performance of the CLFR-hybrid system, an angle range of $0^{\circ}$ to $90^{\circ}$ is considered for both receivers with a focal length of $1500 \mathrm{~mm}$ and a solar field width of $2100 \mathrm{~mm}$. Variations of concentration and utilization ratios with the receiver inclination angle are illustrated in Fig. 19. It is noteworthy that the highest concertation ratio is achieved with horizontal receivers. Both concentration and utilization ratios decrease remarkably as the receiver inclination angle is over $55^{\circ}$. Though more mirrors could be placed in the solar field for receivers at higher inclination angle, the system concentration ratio is lower due to the larger amount of narrow mirrors. For example, the widths of mirror elements in the common tilting array are less than $10 \mathrm{~mm}$ for receivers at an inclination angle of $70^{\circ}$, while much wider mirrors (i.e. $48 \mathrm{~mm}$ ) are involved for receivers placed horizontally. On the other hand, the utilization ratio decreases dramatically with the 
receiver inclination angle. This is due to the fact that the gap between two adjacent mirrors gets much larger as the receiver inclination angle increases. In particular, for vertically orientated receivers, the large inevitable space between the first mirror element and receiver results in the lowest utilization ratio.

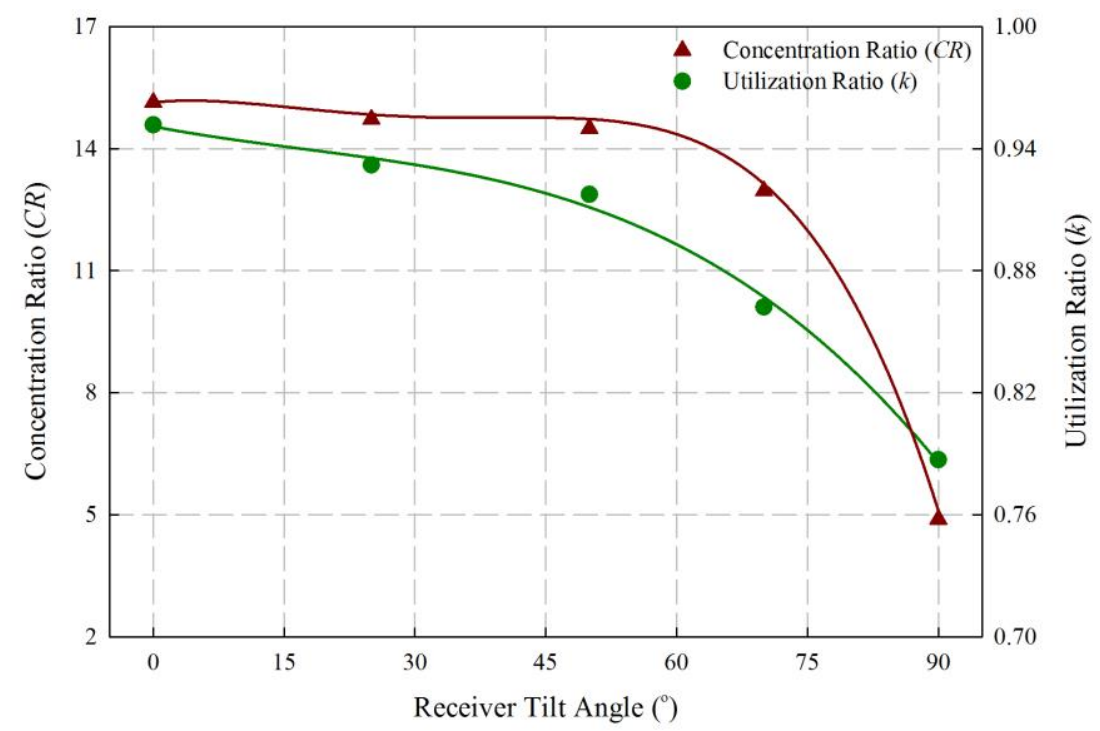

Fig. 19. Variations of concentration and utilization ratios with receiver inclination angle for CLFR-hybrid configurations

\section{Conclusions}

In this paper, detailed geometrical modelling has been established for the CLFR system with polar orientation, which employs flat mirrors and receivers. Based on the numerical modelling, ray tracing simulation and experimental work, a comparative study of concentration characteristics among the LFR, CLFR-complete and CLFR-hybrid systems has been conducted. Moreover, optical design analyses of the CLFR-hybrid system have been performed from different design aspects (i.e. limited solar field area, total number of mirror elements, focal length and receiver inclination angle) based on the numerical simulation results. The following conclusions are drawn: 
(1) The CLFR-hybrid configuration has the highest concentration and utilization ratios compared to the LFR and CLFR-complete systems. Geometrical concentration ratio of 15.14 and ground utilization ratio of 0.95 are obtained for the CLFR-hybrid system with 50 pieces of flat mirror elements, a solar field width of $2100 \mathrm{~mm}$ and a focal length of $1500 \mathrm{~mm}$.

(2) Concentration ratio of the CLFR-hybrid system increases with the total number of mirror elements, while its utilization ratio decreases conversely. The ground usage should be considered to avoid a low utilization ratio while achieving a high concentration ratio.

(3) There exists an optimum mirror array arrangement for the CLFR-hybrid system even though a more densely packed mirror array is favourable. The optimum proportion of alternating mirrors in the central alternating array varies remarkably with the solar field area at a given focal length. For a larger solar field, more mirrors should be accommodated in the common tilting array to achieve a higher concentration ratio.

(4) There is an optimum focal length for the CLFR-hybrid system with limited solar field area when the mirror array arrangement is settled.

(5) Horizontal flat receivers are preferred for the CLFR-hybrid system with better optical performance. The receiver inclination angle over $55^{\circ}$ results in significant reductions in both concentration and utilization ratios.

This paper have presented a generalized methodology for optical design of the CLFR-hybrid system and demonstrated the feasibility of the CLFR-hybrid system with flat mirrors and polar orientation. The work provides progress to the field of concentrated solar power.

\section{Acknowledgements}


The authors gratefully acknowledge the scholarship support from the Faculty of Engineering of the University of Nottingham.

\section{References}

[1] D. Barlev, R. Vidu, P. Stroeve, Innovation in concentrated solar power, Solar Energy Materials and Solar Cells, 95 (2011) 2703-2725.

[2] H. Zhai, Y.J. Dai, J.Y. Wu, R.Z. Wang, L.Y. Zhang, Experimental investigation and analysis on a concentrating solar collector using linear Fresnel lens, Energy Conversion and Management, 51 (2010) 48-55.

[3] F.J. Ruiz-Cabañas, C. Prieto, V. Madina, A.I. Fernández, L.F. Cabeza, Materials selection for thermal energy storage systems in parabolic trough collector solar facilities using high chloride content nitrate salts, Solar Energy Materials and Solar Cells, 163 (2017) 134-147.

[4] D.A. Baharoon, H.A. Rahman, W.Z.W. Omar, S.O. Fadhl, Historical development of concentrating solar power technologies to generate clean electricity efficiently - A review, Renewable and Sustainable Energy Reviews, 41 (2015) 996-1027.

[5] G. Cau, D. Cocco, Comparison of Medium-size Concentrating Solar Power Plants based on Parabolic Trough and Linear Fresnel Collectors, Energy Procedia, 45 (2014) 101-110.

[6] G. Barale, A. Heimsath, P. Nitz, A. Toro, Optical design of a linear Fresnel collector for Sicily, in: 16th SolarPACES International Symposium, Perpignan, France, 2010.

[7] A. Barbón, N. Barbón, L. Bayón, J.A. Otero, Theoretical elements for the design of a small scale Linear Fresnel Reflector: Frontal and lateral views, Solar Energy, 132 (2016) 188-202.

[8] T. Matsushima, T. Setaka, S. Muroyama, Concentrating solar module with horizontal reflector, Solar Energy Materials and Solar Cells, 75 (2003) 603-612. 
[9] V. Sharma, J.K. Nayak, S.B. Kedare, Effects of shading and blocking in linear Fresnel reflector field, Solar Energy, 113 (2015) 114-138.

[10] R. Abbas, M.J. Montes, M. Piera, J.M. Martínez-Val, Solar radiation concentration features in Linear Fresnel Reflector arrays, Energy Conversion and Management, 54 (2012) 133-144.

[11] A. Barbón, N. Barbón, L. Bayón, J.A. Otero, Optimization of the length and position of the absorber tube in small-scale Linear Fresnel Concentrators, Renewable Energy, 99 (2016) 986-995.

[12] T.C. Kandpal, S.S. Mathur, R.N. Singh, A fresnel-winston tandem concentrator system, International Journal of Energy Research, 5 (1981) 77-82.

[13] R. Abbas, J.M. Martínez-Val, Analytic optical design of linear Fresnel collectors with variable widths and shifts of mirrors, Renewable Energy, 75 (2015) 81-92.

[14] Y. Qiu, Y.-L. He, M. Wu, Z.-J. Zheng, A comprehensive model for optical and thermal characterization of a linear Fresnel solar reflector with a trapezoidal cavity receiver, Renewable Energy, 97 (2016) 129-144.

[15] H. Mousazadeh, A. Keyhani, A. Javadi, H. Mobli, K. Abrinia, A. Sharifi, A review of principle and sun-tracking methods for maximizing solar systems output, Renewable and Sustainable Energy Reviews, 13 (2009) 1800-1818.

[16] S. Ozcelik, H. Prakash, R. Challoo, Two-Axis Solar Tracker Analysis and Control for Maximum Power Generation, Procedia Computer Science, 6 (2011) 457-462.

[17] L. Barker, M. Neber, H. Lee, Design of a low-profile two-axis solar tracker, Solar Energy, 97 (2013) 569-576.

[18] J. Shingleton, One-axis trackers—improved reliability, durability, performance and cost reduction, (2008). 
[19] M.J. Montes, C. Rubbia, R. Abbas, J.M. Martínez-Val, A comparative analysis of configurations of linear Fresnel collectors for concentrating solar power, Energy, 73 (2014) 192-203.

[20] D.R. Mills, G.L. Morrison, Compact linear Fresnel reflector solar thermal powerplants, Solar Energy, 68 (2000) 263-283.

[21] J. Chaves, M. Collares-Pereira, Etendue-matched two-stage concentrators with multiple receivers, Journal Name: Solar Energy; Journal Volume: 84; Journal Issue: 2; Other Information: Elsevier Ltd. All rights reserved, (2010) Medium: X; Size: page(s) 196-207.

[22] J. He, Z. Qiu, Q. Li, Y. Zhang, Optical Design of Linear Fresnel Reflector Solar Concentrators, Energy Procedia, 14 (2012) 1960-1966.

[23] Q. Zhang, Theoretical analysis and experimental study of lineat fresnel reflector solar concentrator, in: Engineering Thermophysics, University of Science and Technology of China, 2013.

[24] C. Sharma, A.K. Sharma, S.C. Mullick, T.C. Kandpal, A study of the effect of design parameters on the performance of linear solar concentrator based thermal power plants in India, Renewable Energy, 87 (2016) 666-675.

[25] Y. Qiu, Y.-L. He, Z.-D. Cheng, K. Wang, Study on optical and thermal performance of a linear Fresnel solar reflector using molten salt as HTF with MCRT and FVM methods, Applied Energy, 146 (2015) 162-173.

[26] G. Zhu, Development of an analytical optical method for linear Fresnel collectors, Solar Energy, 94 (2013) 240-252.

[27] M.A. Moghimi, K.J. Craig, J.P. Meyer, A novel computational approach to combine the optical and thermal modelling of Linear Fresnel Collectors using the finite volume method, Solar Energy, 116 (2015) 407-427. 
[28] D. Chemisana, J. Barrau, J.I. Rosell, B. Abdel-Mesih, M. Souliotis, F. Badia, Optical performance of solar reflective concentrators: A simple method for optical assessment, Renewable Energy, 57 (2013) 120-129.

[29] F.J. Pino, R. Caro, F. Rosa, J. Guerra, Experimental validation of an optical and thermal model of a linear Fresnel collector system, Applied Thermal Engineering, 50 (2013) 14631471.

[30] B.S. Negi, S.S. Mathur, T.C. Kandpal, Optical and thermal performance evaluation of a linear fresnel reflector solar concentrator, Solar \& Wind Technology, 7 (1990) 379-392.

[31] S.S. Sahoo, S.M. Varghese, C. Suresh Kumar, S.P. Viswanathan, S. Singh, R. Banerjee, Experimental investigation and computational validation of heat losses from the cavity receiver used in linear Fresnel reflector solar thermal system, Renewable Energy, 55 (2013) $18-23$.

[32] S.S. Mathur, T.C. Kandpal, B.S. Negi, Optical design and concentration characteristics of linear Fresnel reflector solar concentrators-I. Mirror elements of varying width, Energy Conversion and Management, 31 (1991) 205-219.

[33] R. Foster, M. Ghassemi, A. Cota, Solar Energy: Renewable Energy and the Environment, CRC Press, Boca Raton, 2010.

[34] F. Göhring, O. Bender, M. Röger, J. Nettlau, P. Schwarzbözl, Flux density measurement on open volumetric receivers, Proceedings of SolarPACES, (2011) 20-23. 